\title{
Tin-Free and Catalytic Radical Cyclizations
}

\author{
Deborah M. Smith, Mary E. Pulling and Jack R. Norton* \\ Department of Chemistry, Columbia University \\ New York, NY 10025
}

\section{Supporting Information}

\section{Contents:}
I. Synthesis of Dienes
$S-1$
II. Cyclization of Dienes
$S-4$
III. Stereochemical proofs of cyclic products
$S-5$
IV. Bibliography
$S-7$
V. Analytical Data
S-8

General. ${ }^{1} \mathrm{H}$ NMR and ${ }^{13} \mathrm{C}$ NMR spectra were recorded at ambient temperature at $400 \mathrm{MHz}$ and 100 or 75 $\mathrm{MHz}$, respectively, using a Bruker DRX400 or DRX 300 spectrometer. The data are reported as follows: chemical shift in ppm from internal tetramethylsilane on the $\delta$ scale, multiplicity $(\mathrm{br}=$ broad, $\mathrm{s}=$ singlet, $\mathrm{d}=$ doublet, $\mathrm{t}=$ triplet, $\mathrm{q}=$ quartet, $\mathrm{m}=$ multiplet), coupling constants $(\mathrm{Hz})$, and integration. Infrared (IR) spectra were obtained using a Perkin Elmer 2000 FT-IR spectrometer. High resolution mass spectra were acquired on a JOEL JMS-HX110 HF mass spectrometers, and were obtained by peak matching. Liquid chromatography was performed using forced flow (flash chromatography) of the indicated solvent system on Fisher silica gel $\left(\mathrm{SiO}_{2}\right)$ 60 (230-400 mesh). Preparative reverse phase (RP) HPLC was performed on a Grace Vydac C18 Protein \& Peptide column with a Waters 600 Controller; fractions were detected with a Waters 2487 Dual $\lambda$ Absorbance Detector $(218$ and $254 \mathrm{~nm}) . \quad \mathrm{CpCr}(\mathrm{CO})_{3} \mathrm{H}$ was stored and manipulated in an inert nitrogen atmosphere dry box $\left(\mathrm{O}_{2}<1 \mathrm{ppm}\right)$. Reaction mixtures involving $\mathrm{CpCr}(\mathrm{CO})_{3} \mathrm{H}$ were prepared in the drybox. High pressure reactions were carried out in a Fisher-Porter bottle equipped with a pressure gauge, gas inlet, and pressure release valve. All reactions were carried out under an atmosphere of nitrogen in glassware that had been flamedried under a stream of nitrogen. Deuterated benzene $\left(\mathrm{C}_{6} \mathrm{D}_{6}\right)$ was purified by vacuum transfer from sodiumbenzophenone ketyl. Unless otherwise noted, all reagents were commercially obtained and, where appropriate, purified prior to use. THF, $\mathrm{Et}_{2} \mathrm{O}$, toluene, and $\mathrm{CH}_{2} \mathrm{Cl}_{2}$ were dried by filtration through alumina according to the procedure of Grubbs. ${ }^{1}$

\section{Synthesis of Diene Substrates}

\section{A. Diene Substrate (4) Synthetic Scheme.}



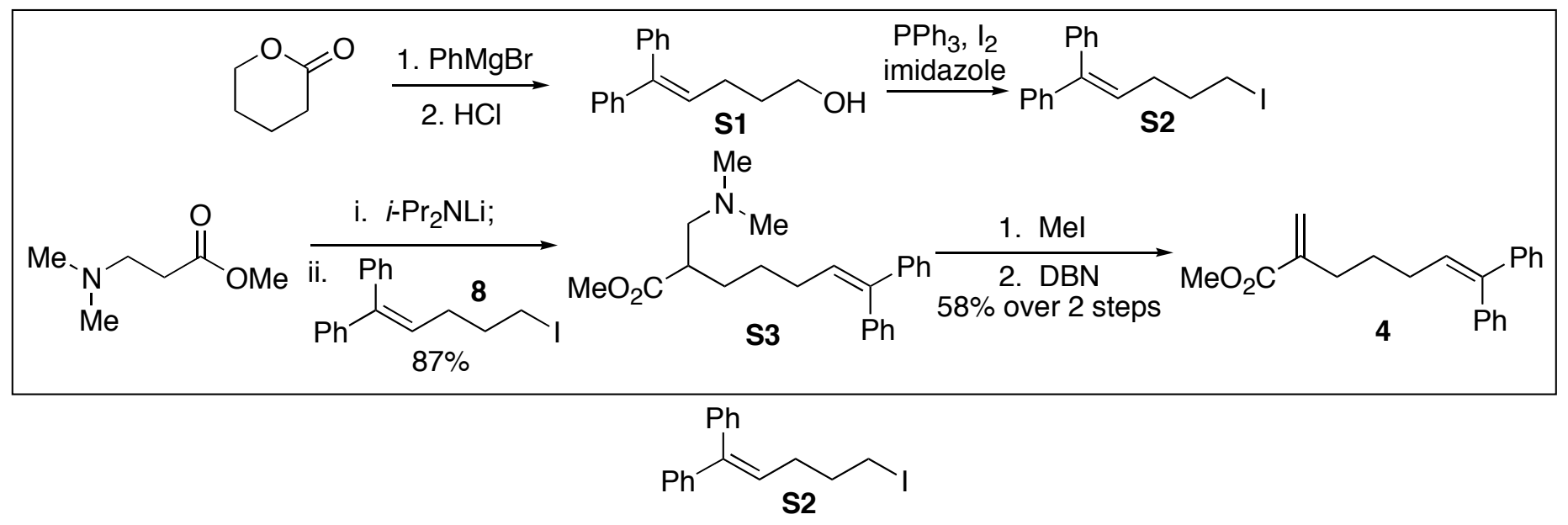

Alkene (S2). To a cooled $\left(0{ }^{\circ} \mathrm{C}\right)$ solution of $\mathrm{PPh}_{3}(3.68 \mathrm{~g}, 14.0 \mathrm{mmol})$ and imidazole $(1.27 \mathrm{~g}, 18.7 \mathrm{mmol})$ in 26 $\mathrm{mL}$ of 3:1 $\mathrm{Et}_{2} \mathrm{O} / \mathrm{CH}_{3} \mathrm{CN}$ was added iodine $(3.56 \mathrm{~g}, 14.0 \mathrm{mmol}){ }^{2}$ After $20 \mathrm{~min}$ at $0{ }^{\circ} \mathrm{C}$, a solution of alcohol $\mathbf{S 1}^{3}$ in $5 \mathrm{~mL}$ of 3:1 Et $\mathrm{E}_{2} / \mathrm{CH}_{3} \mathrm{CN}$ was added dropwise over $10 \mathrm{~min}$. The mixture was warmed to $22^{\circ} \mathrm{C}$ for $12 \mathrm{~h}$. Pentane was added to precipitate triphenylphosphine oxide and the solution was filtered through a pad of silica gel and concentrated in vacuo. ${ }^{4}$ Purification by flash chromatography (2:98-5:95 EtOAc/hexanes) afforded the product as a colorless oil $(3.26 \mathrm{~g}, 91 \%)$ : ${ }^{1} \mathrm{H} \mathrm{NMR}\left(400 \mathrm{MHz}, \mathrm{CDCl}_{3}\right) \delta 7.16-7.40(\mathrm{~m}, 10 \mathrm{H}), 6.03(\mathrm{t}, J=7.6$, $1 \mathrm{H}), 3.15(\mathrm{t}, J=7.0,1 \mathrm{H}), 2.21(\mathrm{q}, J=7.2,2 \mathrm{H}), 1.97$ (quintet, $J=7.2,2 \mathrm{H}) ;{ }^{13} \mathrm{C} \mathrm{NMR}\left(100 \mathrm{MHz}, \mathrm{CDCl}_{3}\right) \delta$ 144.4, 143.7, 141.2, 131.2, 129.6, 129.5, 128.7, 128.5, 128.4, 35.2, 32.0, 7.2; IR (thin film) 3018, 2937, 1594, $1440 \mathrm{~cm}^{-1}$; HRMS (FAB) $\mathrm{m} / \mathrm{z}$ calcd for $\mathrm{C}_{17} \mathrm{H}_{17} \mathrm{I}\left(\mathrm{M}^{+}\right) 348.0375$, found 348. 0380.

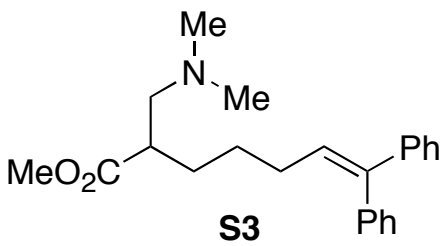

Amine (S3). To a cooled $\left(-78{ }^{\circ} \mathrm{C}\right)$ solution of diisopropylamine $(2.62 \mathrm{~mL}, 18.7 \mathrm{mmol})$ in $75 \mathrm{~mL}$ of THF was added cold $\left(-78{ }^{\circ} \mathrm{C}\right) n$-BuLi $\left(12 \mathrm{~mL}, 18 \mathrm{mmol}, 1.5 \mathrm{M}\right.$ in hexanes). The mixture was stirred at $0{ }^{\circ} \mathrm{C}$ for $30 \mathrm{~min}$ and was again cooled to $-78{ }^{\circ} \mathrm{C}$. A solution of methyl 3-(dimethylamino)propionate $(2.47 \mathrm{~mL}, 17.2 \mathrm{mmol})$ in $40 \mathrm{~mL}$ of THF was cooled to $-78^{\circ} \mathrm{C}$ and added to the reaction mixture by cannula over 3 min. After $45 \mathrm{~min}$, a solution of cold $\left(-78{ }^{\circ} \mathrm{C}\right)$ alkyliodide $\mathbf{S 2}(5.00 \mathrm{~g}, 14.4 \mathrm{mmol})$ and HMPA $(3.00 \mathrm{~mL}, 17.2 \mathrm{mmol})$ in $40 \mathrm{~mL}$ of THF was added to the reaction mixture dropwise by cannula. ${ }^{5}$ After $12 \mathrm{~h}$ at $22{ }^{\circ} \mathrm{C}, 20 \mathrm{~mL}$ of saturated aqueous $\mathrm{NH}_{4} \mathrm{Cl}$ was added. The reaction mixture was extracted with EtOAc $(50 \mathrm{~mL})$. The organic layer was washed with $\mathrm{H}_{2} \mathrm{O}(1 \times 30 \mathrm{~mL})$, saturated aqueous $\mathrm{LiCl}(2 \times 30 \mathrm{~mL})$, dried $\left(\mathrm{Na}_{2} \mathrm{SO}_{4}\right)$, and concentrated in vacuo. The product was isolated by flash chromatography $\left(20: 79: 1-79: 20: 1 \mathrm{EtOAc} /\right.$ hexanes/Et $\left._{3} \mathrm{~N}\right)$ as a colorless oil $(5.05$ $\mathrm{g}, 87 \%):{ }^{1} \mathrm{H}$ NMR $\left(400 \mathrm{MHz}, \mathrm{CDCl}_{3}\right) \delta 7.06-7.30(\mathrm{~m}, 10 \mathrm{H}), 5.96(\mathrm{t}, J=7.6,1 \mathrm{H}), 3.60(\mathrm{~s}, 3 \mathrm{H}), 2.54(\mathrm{~m}, 1 \mathrm{H})$, $2.47(\mathrm{~m}, 1 \mathrm{H}), 2.10(\mathrm{~s}, 6 \mathrm{H}), 2.04(\mathrm{~m}, 3 \mathrm{H}), 1.48(\mathrm{~m}, 1 \mathrm{H}), 1.35(\mathrm{~m}, 3 \mathrm{H}) ;{ }^{13} \mathrm{C} \mathrm{NMR}\left(100 \mathrm{MHz}, \mathrm{CDCl}_{3}\right) \delta 174.9$, 141.7, 140.9, 139.1, 128.9, 128.3, 127.1, 127.0, 126.2, 125.9, 125.8, 61.0, 50.5, 44.7, 43.4, 29.4, 28.6, 26.7; IR (thin film) 2945, 1735, 1443, $1262 \mathrm{~cm}^{-1}$; HRMS (FAB) $\mathrm{m} / \mathrm{z}$ calcd for $\mathrm{C}_{23} \mathrm{H}_{30} \mathrm{NO}_{2}(\mathrm{M}+\mathrm{H})^{+} 352.2277$, found 352.2268 . 


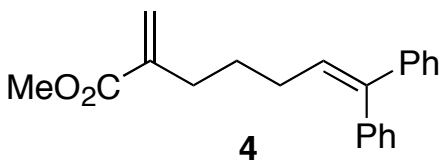

Diene (S4). To a solution of ester S3 (4.378 g, $12.45 \mathrm{mmol})$ in $120 \mathrm{~mL}$ of THF was added iodomethane (1.55 $\mathrm{mL}, 25.9 \mathrm{mmol}$, filtered through basic alumina). ${ }^{6}$ After $12 \mathrm{~h}$ at $22{ }^{\circ} \mathrm{C}$, the solution was concentrated in vacuo to provide a yellow solid. To a solution of this residue in benzene (100 mL) was added DBN (3.02 mL, 25.3 mmol). ${ }^{7}$ After $12 \mathrm{~h}$ at $22{ }^{\circ} \mathrm{C}, \mathrm{H}_{2} \mathrm{O}$ was added and the reaction mixture was extracted with pentane $(3 \times 50 \mathrm{~mL})$. The combined organic layers were washed with $\mathrm{H}_{2} \mathrm{O}$, brine, dried $\left(\mathrm{Na}_{2} \mathrm{SO}_{4}\right)$ and concentrated in vacuo. Purification by flash chromatography (10:89:1-30:69:1 EtOAc/hexanes/Et $\left.{ }_{3} \mathrm{~N}\right)$ provided the product as a colorless oil (2.12 g, 56\%): ${ }^{1} \mathrm{H}$ NMR (400 MHz, $\left.\mathrm{C}_{6} \mathrm{D}_{6}\right) \delta 7.29(\mathrm{~m}, 2 \mathrm{H}), 7.06-7.17(\mathrm{~m}, 8 \mathrm{H}), 6.10(\mathrm{~m}, 1 \mathrm{H}), 6.03$ $(\mathrm{t}, J=7.6,1 \mathrm{H}), 5.13(\mathrm{dd}, J=2.8,1.2,1 \mathrm{H}), 3.36(\mathrm{~s}, 3 \mathrm{H}), 2.23(\mathrm{t}, J=7.6,2 \mathrm{H}), 2.09(\mathrm{q}, J=7.6,2 \mathrm{H}), 1.54$ (quintet, $J=7.6,2 \mathrm{H}) ;{ }^{13} \mathrm{C} \mathrm{NMR}\left(100 \mathrm{MHz} \mathrm{CDCl}_{3}\right) \delta 169.0,144.1,143.4,141.7,141.5,131.2,130.7,129.5$, $129.4,128.5,128.21,128.16,126.1,53.1,32.8,30.6,30.1$; IR (thin film) $2945,1720,1628,1439 \mathrm{~cm}^{-1}$. HRMS (FAB) $m / z$ calcd for $\mathrm{C}_{21} \mathrm{H}_{23} \mathrm{O}_{2}(\mathrm{M}+\mathrm{H})^{+} 307.1698$, found 307.1702.

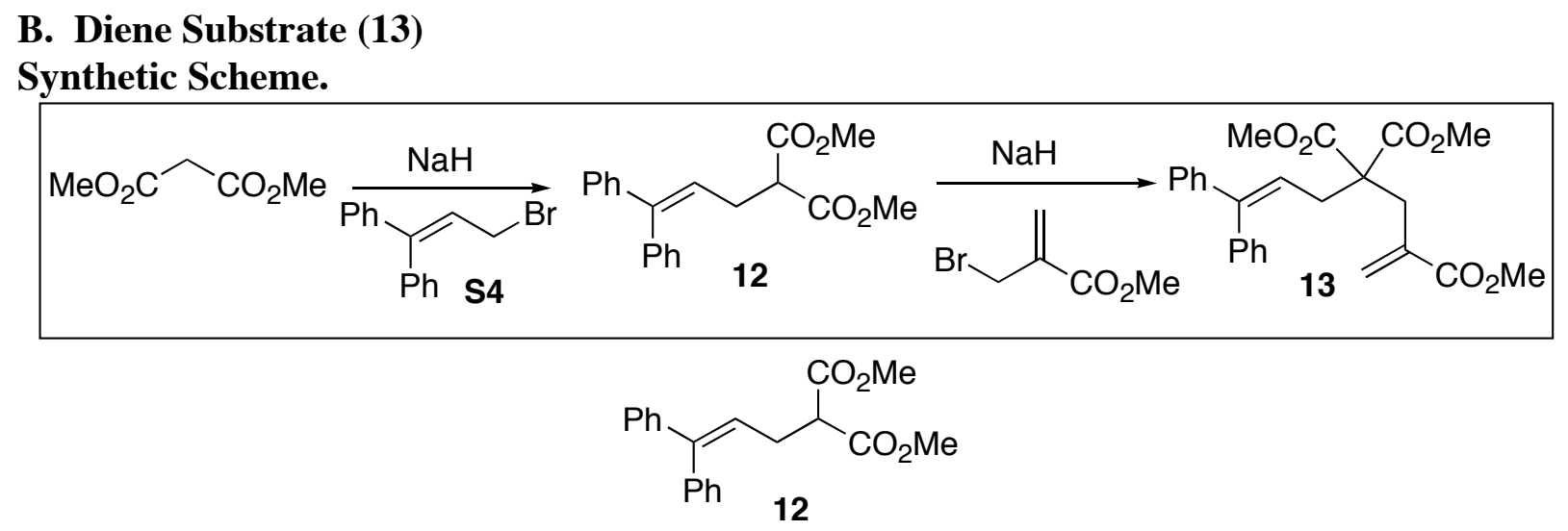

Alkene (12). To a cooled $\left(0{ }^{\circ} \mathrm{C}\right)$ solution of dimethyl malonate $(5.00 \mathrm{~mL}, 43.6 \mathrm{mmol})$ in $125 \mathrm{~mL}$ of THF was added $\mathrm{NaH}(0.900 \mathrm{~g}, 35.8 \mathrm{mmol}, 95 \%) .{ }^{8}$ The mixture was stirred at $0{ }^{\circ} \mathrm{C}$ for $2 \mathrm{~h}$. The reaction mixture was added with a cannula to a $0{ }^{\circ} \mathrm{C}$ solution of 3-bromo-1,1-diphenyl-1-propene $\mathbf{S} 4^{9}$ (7.87 $\left.\mathrm{g}, 28.8 \mathrm{mmol}\right)$ in $45 \mathrm{~mL}$ of THF. After $30 \mathrm{~min}$ at $22{ }^{\circ} \mathrm{C}$, the reaction mixture was treated with saturated aqueous $\mathrm{NH}_{4} \mathrm{Cl}(60 \mathrm{~mL})$, extracted with EtOAc $(2 \times 100 \mathrm{~mL})$, dried $\left(\mathrm{Na}_{2} \mathrm{SO}_{4}\right)$, and concentrated in vacuo. The product was isolated by flash chromatography (4:1 hexanes/EtOAc) as a yellow oil: ${ }^{1} \mathrm{H}$ NMR $\left(400 \mathrm{MHz}, \mathrm{CDCl}_{3}\right) \delta 7.30-7.34(\mathrm{~m}, 4 \mathrm{H})$, 7.13-7.24 (m, 6H), $5.97(\mathrm{t}, J=7.6,1 \mathrm{H}), 3.69(\mathrm{~s}, 6 \mathrm{H}), 3.46(\mathrm{t}, J=7.6,1 \mathrm{H}), 2.72(\mathrm{t}, J=7.6,2 \mathrm{H}) ;{ }^{1} \mathrm{H}$ NMR $(400$ $\left.\mathrm{MHz}, \mathrm{C}_{6} \mathrm{D}_{6}\right) \delta 7.25-7.26(\mathrm{~d}, 2 \mathrm{H}), 7.02-7.13(\mathrm{~m}, 8 \mathrm{H}), 6.11(\mathrm{t}, J=7.4,1 \mathrm{H}), 3.42(\mathrm{t}, J=7.4,1 \mathrm{H}), 3.21(\mathrm{~s}, 6 \mathrm{H})$, $2.88(\mathrm{t}, J=7.4,2 \mathrm{H}) ;{ }^{13} \mathrm{C}$ NMR $\left(75 \mathrm{MHz} \mathrm{CDCl}_{3}\right) \delta 169.6,144.9,142.6,139.7,130.1,128.7,128.5,127.8$, 127.7, 124.6, 52.9, 52.2, 29.3; IR (thin film) 3026, 2955, 1740, 1494, 1440, 1342, 1234, $1158 \mathrm{~cm}^{-1}$; HRMS (FAB) $m / z$ calcd for $\mathrm{C}_{20} \mathrm{H}_{20} \mathrm{O}_{4}\left(\mathrm{M}^{+}\right) 324.1362$, found 324.1373.

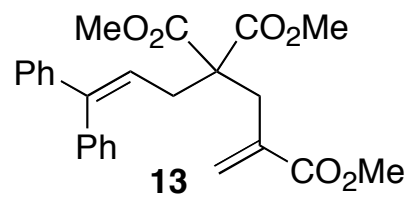

Diene (13). To a cooled $\left(0^{\circ} \mathrm{C}\right)$ solution of dimethyl 2-(3,3-diphenylallyl)malonate $(2.6 \mathrm{~g}, 8.0 \mathrm{mmol})$ in $80 \mathrm{~mL}$ of THF was added $\mathrm{NaH}(0.22 \mathrm{~g}, 8.7 \mathrm{mmol}, 95 \%)$. After $2 \mathrm{~h}$ at $22{ }^{\circ} \mathrm{C}$, the reaction mixture was cooled to $0{ }^{\circ} \mathrm{C}$ and 2-bromomethylacrylic acid $(1.0 \mathrm{~mL}, 8.1 \mathrm{mmol}, 97 \%)$ was added. The solution was heated to reflux. After 
$6 \mathrm{~h}$, the reaction mixture was cooled $\left(22^{\circ} \mathrm{C}\right)$ and treated with saturated aqueous $\mathrm{NH}_{4} \mathrm{Cl}(40 \mathrm{~mL})$, and extracted with EtOAc $(2 \times 75 \mathrm{~mL})$. The combined organic layers were dried $\left(\mathrm{Na}_{2} \mathrm{SO}_{4}\right)$, and concentrated in vacuo. The product was isolated by flash chromatography (5:1 hexanes/EtOAc) as a while solid $(2.8 \mathrm{~g}, 83 \%):{ }^{1} \mathrm{H}$ NMR $\left(400 \mathrm{MHz}, \mathrm{CDCl}_{3}\right) \delta 7.28-7.36(\mathrm{~m}, 3 \mathrm{H}), 7.10-7.23(\mathrm{~m}, 6 \mathrm{H}), 6.16(\mathrm{~s}, 1 \mathrm{H}), 5.91(\mathrm{t}, J=7.2,1 \mathrm{H}), 5.51(\mathrm{~s}, 1 \mathrm{H})$, 3.65, (s, 3H), $3.64(\mathrm{~s}, 6 \mathrm{H}), 3.01(\mathrm{~s}, 2 \mathrm{H}), 2.73(\mathrm{~d}, J=7.2,2 \mathrm{H}) ;{ }^{1} \mathrm{H}$ NMR $\left(400 \mathrm{MHz}, \mathrm{C}_{6} \mathrm{D}_{6}\right) \delta 7.34(\mathrm{~d}, J=6.8,2 \mathrm{H})$, 6.95-7.09 (m, 8H), $6.25(\mathrm{t}, J=7.2,1 \mathrm{H}), 6.12(\mathrm{~s}, 1 \mathrm{H}), 5.36(\mathrm{~s}, 1 \mathrm{H}), 3.31(\mathrm{~s}, 6 \mathrm{H}), 3.30(\mathrm{~s}, 2 \mathrm{H}), 3.26(\mathrm{~s}, 3 \mathrm{H}), 3.06$ $(\mathrm{d}, J=7.2,2 \mathrm{H}) ;{ }^{13} \mathrm{C}$ NMR $\left(75 \mathrm{MHz}, \mathrm{CDCl}_{3}\right) \delta 171.4,167.6,145.5,143.0,139.7,135.9,130.2,129.7,128.6$, 128.5, 127.9, 127.7, 123.1, 58.1, 52.8, 52.3. 34.4, 33.0; IR (thin film) 2953, 1732, 1441, 1288, $1200,1155 \mathrm{~cm}^{-1}$; HRMS (FAB) $m / z$ calcd for $\mathrm{C}_{25} \mathrm{H}_{26} \mathrm{O}_{6}(\mathrm{M}+\mathrm{H})^{+} 423.1807$, found 423.1817 .

\section{Cyclization of Diene Substrates}

General Procedure for $\mathbf{C p C r}(\mathbf{C O})_{3} \mathbf{H}-$ Mediated Cyclization: $\mathrm{CpCr}(\mathrm{CO})_{3} \mathrm{H}$ was synthesized according to published procedures ${ }^{10}$ and sublimed prior to use. A stock solution of $\mathrm{CpCr}(\mathrm{CO})_{3} \mathrm{H}$ in $\mathrm{C}_{6} \mathrm{D}_{6}(0.02-0.04 \mathrm{M})$ was stirred under $30 \mathrm{psi}$ of $\mathrm{H}_{2}$. After $30 \mathrm{~min}$, a solution of diene substrate and hexamethylcyclotrisiloxane in $\mathrm{C}_{6} \mathrm{D}_{6}(0.13 \mathrm{M}$ in substrate) was added to the reaction mixture. The reaction mixture was pressurized to $30 \mathrm{psi}$ of $\mathrm{H}_{2}$. After NMR analysis indicated that the starting material was consumed, the reaction mixture was filtered through a silica plug and concentrated in vacuo. NMR concentrations were determined from integration vs the hexamethylcyclotrisiloxane standard.

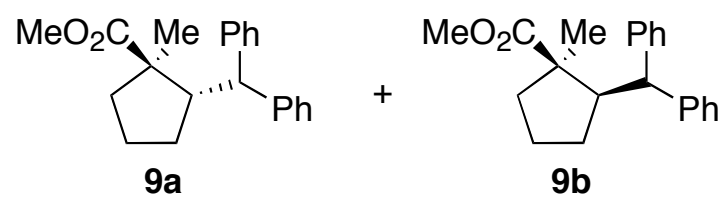

Carbocycle (9). The standard cyclization procedure was followed with diene 4 (0.328 g, $1.07 \mathrm{mmol})$ and $\mathrm{CpCr}(\mathrm{CO})_{3} \mathrm{H}(3.6 \mathrm{~mL}, 0.071 \mathrm{mmol}, 0.020 \mathrm{M})$ and heated to $50{ }^{\circ} \mathrm{C}$ for $4 \mathrm{~d} .{ }^{1} \mathrm{H}$ NMR spectroscopic analysis of the unpurified product showed $9(62 \%)$ as a pair of diastereomers in an 62:38 ratio of diastereomers, hydrogenation product 6 (26\%) and isomerization product $10(12 \%)$. Purification by flash chromatography did not adequately separate the products. The products were separable in small amounts by preparative RP-HPLC.

Major 1,2-trans isomer (9a): ${ }^{1} \mathrm{H} \mathrm{NMR}\left(400 \mathrm{MHz} \mathrm{CDCl}_{3}\right) \delta 7.07-7.35(\mathrm{~m}, 10 \mathrm{H}), 3.70(\mathrm{~d}, J=11.6,1 \mathrm{H}), 3.41$ $(\mathrm{td}, J=11.6,7.6,1 \mathrm{H}), 3.20(\mathrm{~s}, 3 \mathrm{H}), 2.17(\mathrm{~m}, 1 \mathrm{H}), 1.57-1.77(\mathrm{~m}, 4 \mathrm{H}), 1.30(\mathrm{~m}, 1 \mathrm{H}), 1.15(\mathrm{~s}, 3 \mathrm{H}) ;{ }^{13} \mathrm{C}$ NMR $(100$ $\left.\mathrm{MHz}_{,} \mathrm{CDCl}_{3}\right) \delta 179.7,146.4,144.4,129.8,129.7,129.5,129.0,127.6,127.4,56.1,52.7,51.8,42.9,32.2,31.0$, 23.3, 18.2; IR (thin film) 3026, 2945, 1724, 1494, $1252 \mathrm{~cm}^{-1}$; HRMS (FAB) $\mathrm{m} / \mathrm{z}$ calcd. $\mathrm{C}_{21} \mathrm{H}_{24} \mathrm{O}_{2}$ for $\left(\mathrm{M}^{+}\right)$ 308.1776, found 308.1776.

Minor 1,2-cis isomer (9b): ${ }^{1} \mathrm{H}$ NMR $\left(400 \mathrm{MHz} \mathrm{CDCl}_{3}\right) \delta 7.19-7.31(\mathrm{~m}, 8 \mathrm{H}), 7.09(\mathrm{~m}, 2 \mathrm{H}), 3.97(\mathrm{~d}, J=11.6$, $1 \mathrm{H}), 3.49(\mathrm{~s}, 3 \mathrm{H}), 2.72(\mathrm{ddd}, J=11.4,10.3,7.0,1 \mathrm{H}), 2.16(\mathrm{~m}, 1 \mathrm{H}), 1.72(\mathrm{~m}, 2 \mathrm{H}), 1.50-1.63(\mathrm{~m}, 3 \mathrm{H}), 0.91(\mathrm{~s}$, $3 \mathrm{H}) ;{ }^{13} \mathrm{C} \mathrm{NMR}\left(100 \mathrm{MHz}, \mathrm{CDCl}_{3}\right) \delta 177.8,145.8,144.3,128.8,128.7,128.5,128.3,126.7,126.4,55.2,52.1$, 51.6, 42.1, 34.0, 26.3, 23.4; IR (thin film) 3026, 2946, 1721, 1595, $1448 \mathrm{~cm}^{-1}$; HRMS (FAB) $\mathrm{m} / \mathrm{z}$ calcd. $\mathrm{C}_{21} \mathrm{H}_{24} \mathrm{O}_{2}$ for $\left(\mathrm{M}^{+}\right)$308.1776, found 308.1771.<smiles>CC(=O)C(C)CCCC=C(c1ccccc1)c1ccccc1</smiles> 
Hydrogenation Product (6). ${ }^{1} \mathrm{H}$ NMR $\left(400 \mathrm{MHz}^{\mathrm{CDCl}}{ }_{3}\right) \delta 7.15-7.37(\mathrm{~m}, 10 \mathrm{H}), 6.05(\mathrm{t}, J=7.6,1 \mathrm{H}), 3.65(\mathrm{~s}$, $3 \mathrm{H}), 2.40$ (sextet, $J=7.0,1 \mathrm{H}), 2.11(\mathrm{q}, J=7.6,2 \mathrm{H}), 1.65(\mathrm{~m}, 1 \mathrm{H}), 1.42(\mathrm{~m}, 3 \mathrm{H}), 1.12(\mathrm{~d}, J=6.8,3 \mathrm{H}) ;{ }^{13} \mathrm{C} \mathrm{NMR}$ $\left(100 \mathrm{MHz}, \mathrm{CDCl}_{3}\right) \delta 178.5,144.0,143.2,141.5,131.2,130.8,129.5,129.4,128.5,128.21,128.16,52.8,40.6$, $34.7,30.9,28.9,18.4$; IR (thin film) $3025,2937,1734,1440 \mathrm{~cm}^{-1}$; HRMS (FAB) $\mathrm{m} / \mathrm{z}$ calcd. $\mathrm{C}_{21} \mathrm{H}_{24} \mathrm{O}_{2}$ for $\left(\mathrm{M}^{+}\right)$ 308.1776 , found 308.1779 .

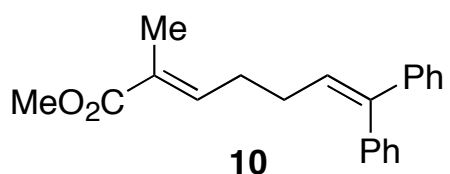

Isomerization Product (10). ${ }^{1} \mathrm{H}$ NMR $\left(400 \mathrm{MHz}, \mathrm{CDCl}_{3}\right) \delta 7.18-7.36(\mathrm{~m}, 10 \mathrm{H}), 6.76(\mathrm{~m}, 1 \mathrm{H}), 6.10(\mathrm{t}, J=7.2$, $1 \mathrm{H}), 3.75(\mathrm{~s}, 3 \mathrm{H}), 2.30(\mathrm{~m}, 4 \mathrm{H}), 1.85(\mathrm{~s}, 3 \mathrm{H}) ;{ }^{13} \mathrm{C} \mathrm{NMR}\left(100 \mathrm{MHz}, \mathrm{CDCl}_{3}\right) \delta 168.5,142.9,142.5,141.6,140.1$, 129.9, 128.4, 128.2, 127.3, 127.2, 51.8, 29.1, 28.9, 12.6; IR (thin film) 3018, 2944, 1712, 1440, $1263 \mathrm{~cm}^{-1}$; HRMS (FAB) $\mathrm{m} / \mathrm{z}$ calcd. $\mathrm{C}_{21} \mathrm{H}_{24} \mathrm{O}_{2}$ for $(\mathrm{M}+\mathrm{H})^{+} 307.1698$, found 307.1688 .

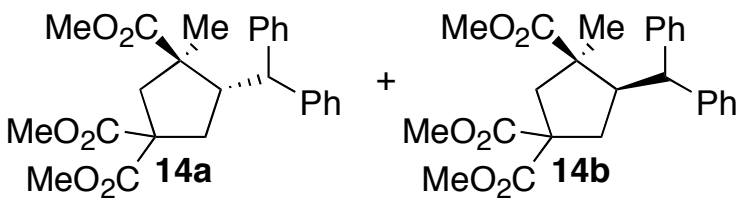

Carbocycle (14). The standard cyclization procedure $(0.30 \mathrm{M}$ in substrate 14) was followed with diene 12 $(0.189 \mathrm{~g}, 0.447 \mathrm{mmol})$ and $\mathrm{CpCr}(\mathrm{CO})_{3} \mathrm{H}(0.75 \mathrm{~mL}, 0.030 \mathrm{mmol}, 0.040 \mathrm{M})$ and heated to $50{ }^{\circ} \mathrm{C}$ for $1.5 \mathrm{~d}$. ${ }^{1} \mathrm{H}$ NMR spectroscopic analysis of the unpurified product showed $14(>95 \%)$ as a 1:1 mixture of diastereomers. Purification by flash chromatography did not adequately separate the products. The products were separable in small amounts by preparative RP-HPLC.

1,2-trans isomer (14a): ${ }^{1} \mathrm{H}$ NMR $\left(400 \mathrm{MHz}, \mathrm{C}_{6} \mathrm{D}_{6}\right) \delta 7.27(\mathrm{~d}, J=7.2,2 \mathrm{H}), 7.20(\mathrm{~d}, J=7.2,2 \mathrm{H}), 6.87-7.07$ (m, $6 \mathrm{H}), 3.93(\mathrm{~m}, 1 \mathrm{H}), 3.67(\mathrm{~d}, J=11.6,1 \mathrm{H}), 3.28(\mathrm{~s}, 3 \mathrm{H}), 3.24(\mathrm{~s}, 3 \mathrm{H}), 3.21(\mathrm{~d}, J=14,1 \mathrm{H}), 2.96(\mathrm{~s}, 3 \mathrm{H}), 2.81(\mathrm{dd}$, $J=14,6.8,1 \mathrm{H}), 2.73(\mathrm{~d}, J=14,1 \mathrm{H}), 2.14(\mathrm{dd}, J=14,12.8,1 \mathrm{H}), 1.32(\mathrm{~s}, 3 \mathrm{H}) ;{ }^{13} \mathrm{C} \mathrm{NMR}\left(75 \mathrm{MHz} \mathrm{CDCl}_{3}\right) \delta$ 176.9, 173.0, 172.8, 144.3, 142.5, 129.1, 128.6, 128.6, 127.9, 126.9, 126.8, 57.7, 54.1, 53.3, 52.1, 51.0, 48.2, 39.4, 17.6; IR (thin film) 2953, 1738, 1732, 1494, 1454, 1435, 1264, $1247 \mathrm{~cm}^{-1}$; HRMS (FAB) $\mathrm{m} / \mathrm{z}$ calcd for $\mathrm{C}_{25} \mathrm{H}_{28} \mathrm{O}_{2}\left(\mathrm{M}^{+}\right) 425.1964$, found 425.1981 .

1,2-cis isomer (9b): ${ }^{1} \mathrm{H}$ NMR $\left(400 \mathrm{MHz}, \mathrm{CDCl}_{3}\right) \delta$ 7.09-7.25 (m, 10H), $3.88(\mathrm{~d}, J=11.2,1 \mathrm{H}), 3.71(\mathrm{~s}, 3 \mathrm{H})$, $3.67(\mathrm{~s}, 1 \mathrm{H}), 3.65(\mathrm{~s}, 3 \mathrm{H}), 2.98(\mathrm{~m}, 1 \mathrm{H}), 2.95(\mathrm{~d}, J=14.4,1 \mathrm{H}), 2.23-2.34(\mathrm{~m}, 2 \mathrm{H}), 2.20(\mathrm{~d}, J=14.4,1 \mathrm{H}), 0.75$ $(\mathrm{s}, 3 \mathrm{H}) ;{ }^{1} \mathrm{H}$ NMR $\left(400 \mathrm{MHz}, \mathrm{C}_{6} \mathrm{D}_{6}\right) \delta 7.30(\mathrm{~d}, J=7.2,2 \mathrm{H}), 7.20(\mathrm{~d}, J=7.2,2 \mathrm{H}), 6.93-7.08(\mathrm{~m}, 6 \mathrm{H}), 4.23(\mathrm{~d}, J=$ $11.6,1 \mathrm{H}), 3.34(\mathrm{~s}, 3 \mathrm{H}), 3.31(\mathrm{~s}, 3 \mathrm{H}), 3.31(\mathrm{~d}, J=14,1 \mathrm{H}), 3.26(\mathrm{~s}, 3 \mathrm{H}), 3.10(\mathrm{~m}, 1 \mathrm{H}), 2.72(\mathrm{~m}, 2 \mathrm{H}), 2.37(\mathrm{~d}, J=$ $14,1 \mathrm{H}), 0.87(\mathrm{~s}, 3 \mathrm{H}) ;{ }^{13} \mathrm{C} \mathrm{NMR}\left(100 \mathrm{MHz} \mathrm{CDCl}_{3}\right.$, distinctive peaks) $\delta 176.2,173.7,172.1,144.5,143.4,128.8$, $128.4,127.8,126.7,126.5,57.5,54.2,53.5,53.0,52.8,52.2,51.6,47.8,41.0,24.9,17.5$; IR (thin film) 2953, $1738,1732,1599,1495,1453,1435,1265 \mathrm{~cm}^{-1}$; HRMS (FAB) $\mathrm{m} / z$ calcd for $\mathrm{C}_{25} \mathrm{H}_{28} \mathrm{O}_{2}\left(\mathrm{M}^{+}\right) 425.1964$, found 425.1957.

\section{Stereochemical proofs of cyclic products}

A. The stereochemistry of cyclopentane $\mathbf{9}$ was determined by analysis of nOe data:

Relevant DPFGSE-nOe data (mixing time $0.5 \mathrm{~s}$ ): (the peaks in the ${ }^{1} \mathrm{H}$ NMR spectra were assigned using ${ }^{1} \mathrm{H} /{ }^{1} \mathrm{H}$ COSY, ${ }^{1} \mathrm{H}$ NMR chemical shifts, and ${ }^{1} \mathrm{H}$ NMR coupling constants) 


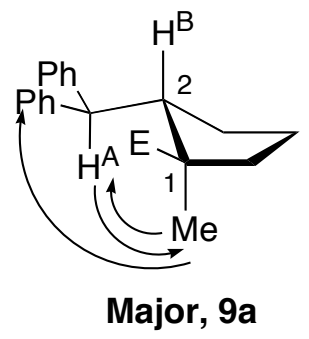

\section{Cyclopentane 9a (1,2-trans, major)}

nOe experiments were performed for major 9a using a pure sample of the isomer in $\mathrm{CDCl}_{3}$

$\mathbf{H}^{\mathbf{A}}$ irradiated: $\mathrm{Me}(3.3 \%)$

Me irradiated: $\mathrm{H}^{\mathrm{A}}(1.75 \%), \mathrm{Ph}(0.61 \%), \mathrm{H}^{\mathrm{B}}(0.3 \%)$

Note: The observation of a large nOe enhancement between $\mathrm{H}^{\mathrm{A}}$ and Me suggests a 1,2trans configuration. ${ }^{11}$

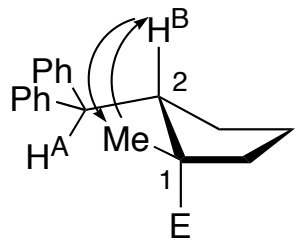

minor, 9b

\section{Cyclopentane 9b (1,2-cis, minor)}

nOe experiments were performed for minor $\mathbf{9 b}$ using a pure sample of the isomer in $\mathrm{CDCl}_{3}$

$\mathbf{H}^{\mathbf{B}}$ irradiated: $\mathrm{Me}(2.3 \%)$

Me irradiated: $\mathrm{H}^{\mathrm{B}}(1.3 \%)$

Note: The observation of large nOe enhancements between $\mathrm{H}^{\mathrm{B}}$ and Me suggests a 1,2-cis configuration. ${ }^{11}$

B. The stereochemistry of cyclopentane $\mathbf{1 4}$ was determined by analysis of nOe data:

Relevant DPFGSE-nOe data (mixing time 2.0 s): (the peaks in the ${ }^{1} \mathrm{H}$ NMR spectra were assigned using ${ }^{1} \mathrm{H} /{ }^{1} \mathrm{H}$ COSY, ${ }^{1} \mathrm{H}$ NMR chemical shifts, and ${ }^{1} \mathrm{H}$ NMR coupling constants)

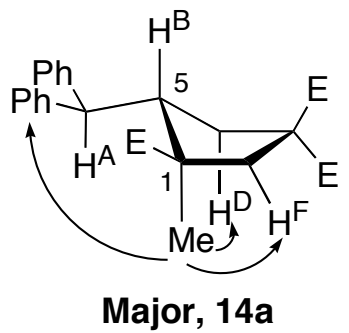

\section{Cyclopentane 14a (1,2-trans, major)}


nOe experiments were performed for major $\mathbf{1 4 a}$ using a pure sample of the isomer in $\mathrm{CDCl}_{3}$

Me irradiated: $\mathrm{H}^{\mathrm{D}}(1.6 \%), \mathrm{H}^{\mathrm{F}}(1.6 \%), \mathrm{Ph}(1.0 \%)$

$\mathbf{H}^{\mathrm{D}}$ irradiated: $\mathrm{Me}(3.1 \%), \mathrm{H}^{\mathrm{C}}(3.6 \%)$

Note: The observation of nOe between $\mathrm{Me}$ and $\mathrm{Ph}$ along with $\mathrm{Me}$ and $\mathrm{H}^{\mathrm{D}}$ suggests a 1,2trans configuration. ${ }^{11}$

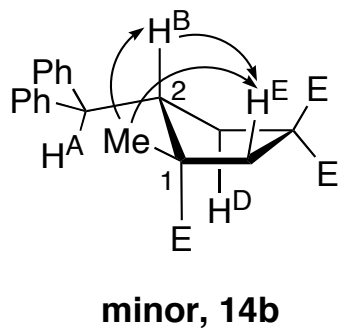

Cyclopentane 14b (1,2-cis, minor)

nOe experiments were performed for minor $\mathbf{1 4 b}$ using a pure sample of the isomer in $\mathrm{C}_{6} \mathrm{D}_{6}$

Me irradiated: $\mathrm{H}^{\mathrm{E}}(1.4 \%), \mathrm{H}^{\mathrm{B}}(1.4 \%)$

$\mathbf{H}^{\mathbf{E}}$ irradiated: $\mathrm{Me}(3.0 \%), \mathrm{H}^{\mathrm{B}}(0.9 \%)$

$\mathbf{H}^{\mathbf{B}}$ irradiated: $\mathrm{H}^{\mathrm{E}}(1.4 \%), \mathrm{Ph}(7.7 \%)$

Note: The observation of nOe between $\mathrm{Me}$ and $\mathrm{H}^{\mathrm{E}}$ along with a large nOe enhancement between $\mathrm{Me}$ and $\mathrm{H}^{\mathrm{E}}$ suggests a 1,2-cis configuration. ${ }^{11}$

\section{Bibliography}

1. Pangborn, A. B.; Giardello, M. A.; Grubbs, R. H.; Rosen, R. K.; Timmers, F. J. Organometallics 1996, 15, $1518-1520$.

2. Wang, Y.; Dong, X.; Larock, R. C. J. Org. Chem. 2003, 68, 3090-3098.

3. Lehmann, J.; Marquardt, N. Liebigs Ann. Chem. 1988, 827-831.

4. Lomberget, T.; Bouyssi, D.; Balme, G. Synthesis 2005, 311-329.

5. Yu, L.-C.; Helquist, P. J. Org. Chem. 1981, 46, 4536-4541.

6. Langlois, N.; Nguyen, B. K. L. J. Org. Chem. 2004, 69, 7558-7564.

7. Nickon, A.; Rodriguez, A. D.; Ganguly, R.; Shirhatti, V. J. Org. Chem. 1985, 50, 2767-2777.

8. Hayashi, Y.; Gotoh, H.; Tamura, T.; Yamaguchi, H.; Masui, R.; Shoji, M. J. Am. Chem. Soc. 2005, 127, $16028-16029$.

9. Davis, M. A.; Herr, F.; Thomas, R. A.; Charest, M. P. J. Med. Chem. 1967, 10, 627-635.

10. Keppie, S. A.; Lappert, M. F. J. Organomet. Chem. 1969, 19, 5-6.

11. Bernstein, M. A.; Morton, H. E.; Guindon, Y. J. Chem. Soc., Perkin Trans. 2 1986, 1155-1163. 


\section{Analytical Data}

A. ${ }^{1} \mathrm{H}$ NMR spectra of unpurified isomer ratios

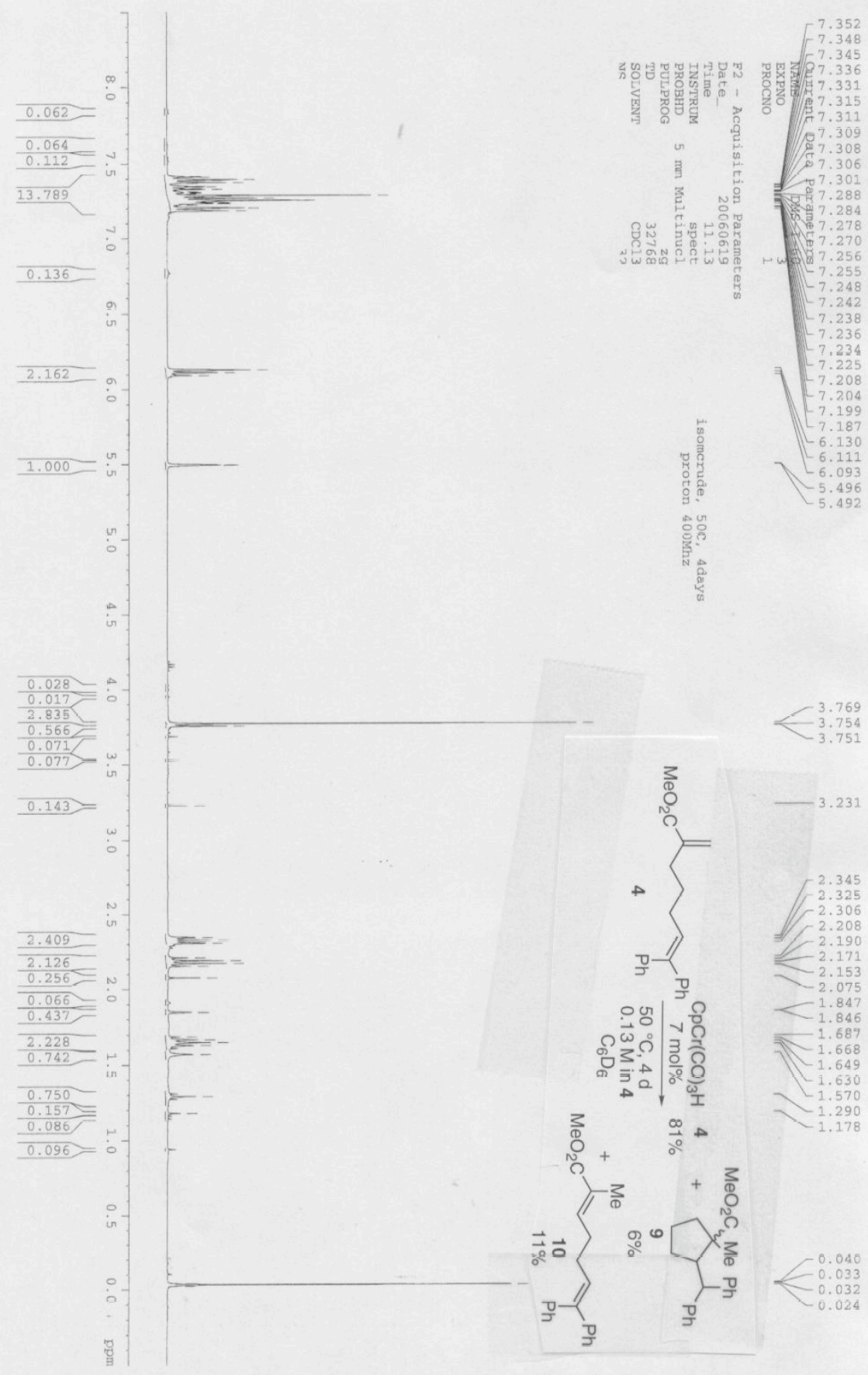




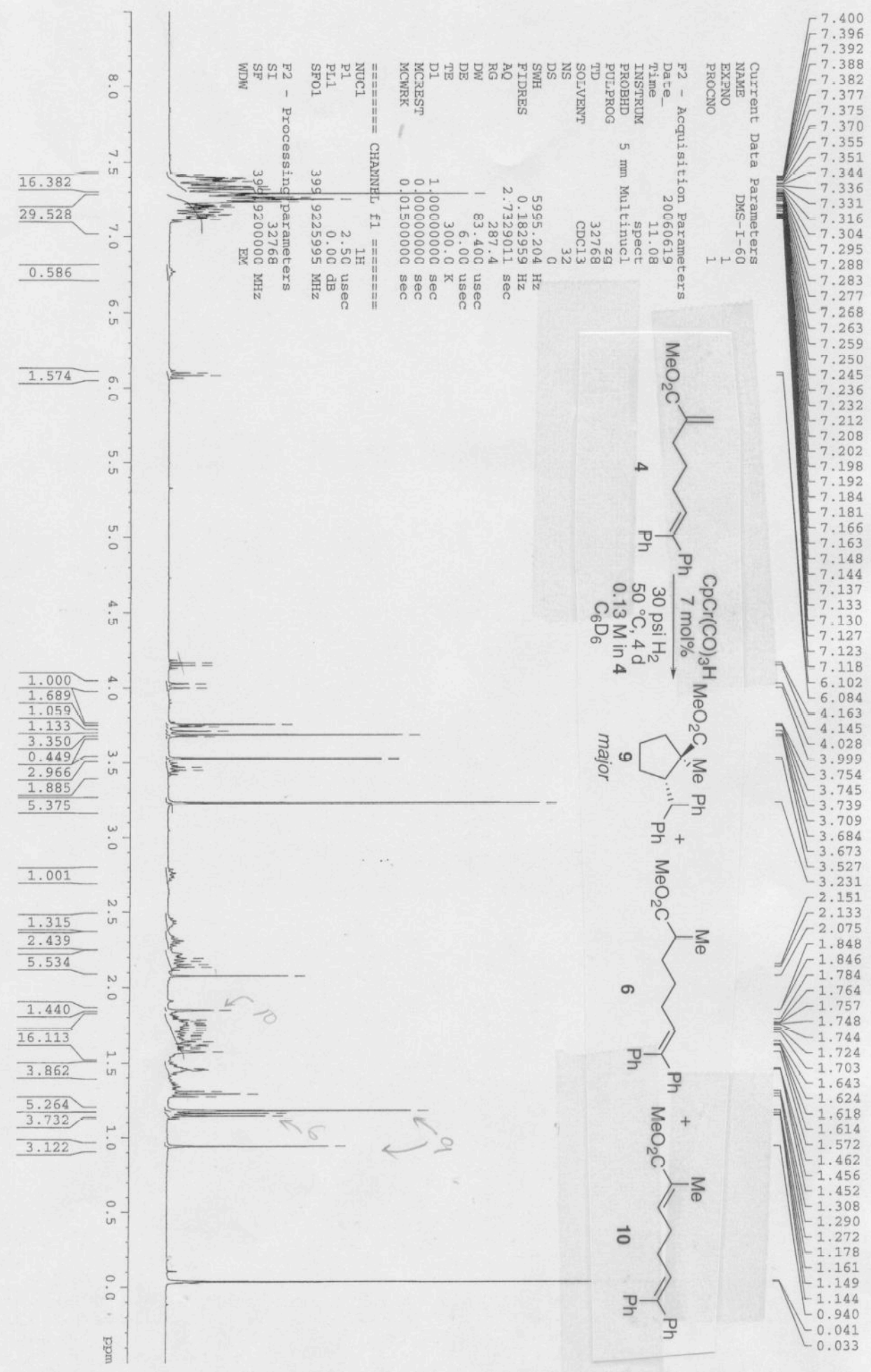




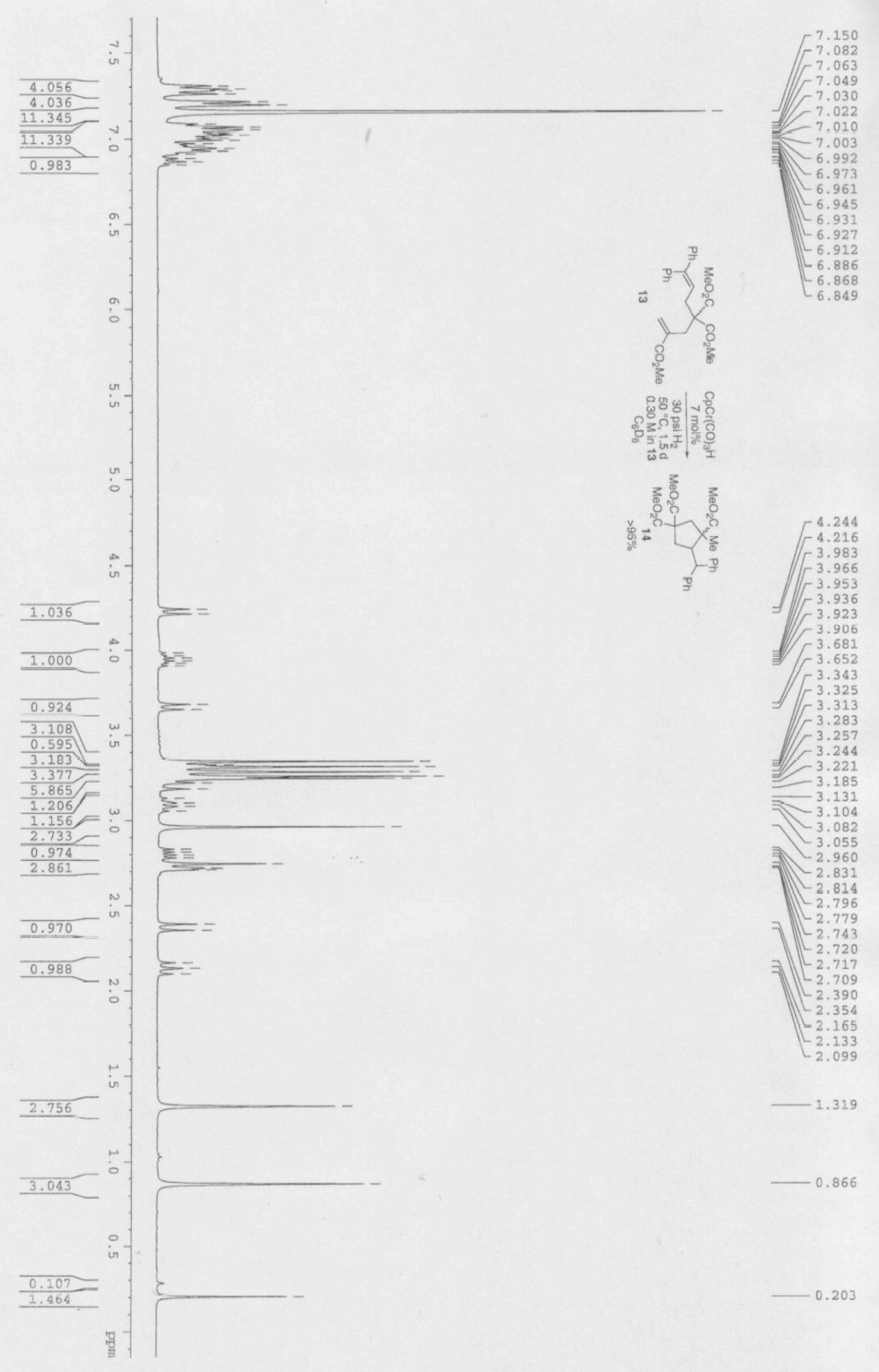


B. ${ }^{1} \mathrm{H}$ and ${ }^{13} \mathrm{C}$ NMR spectra of selected compounds

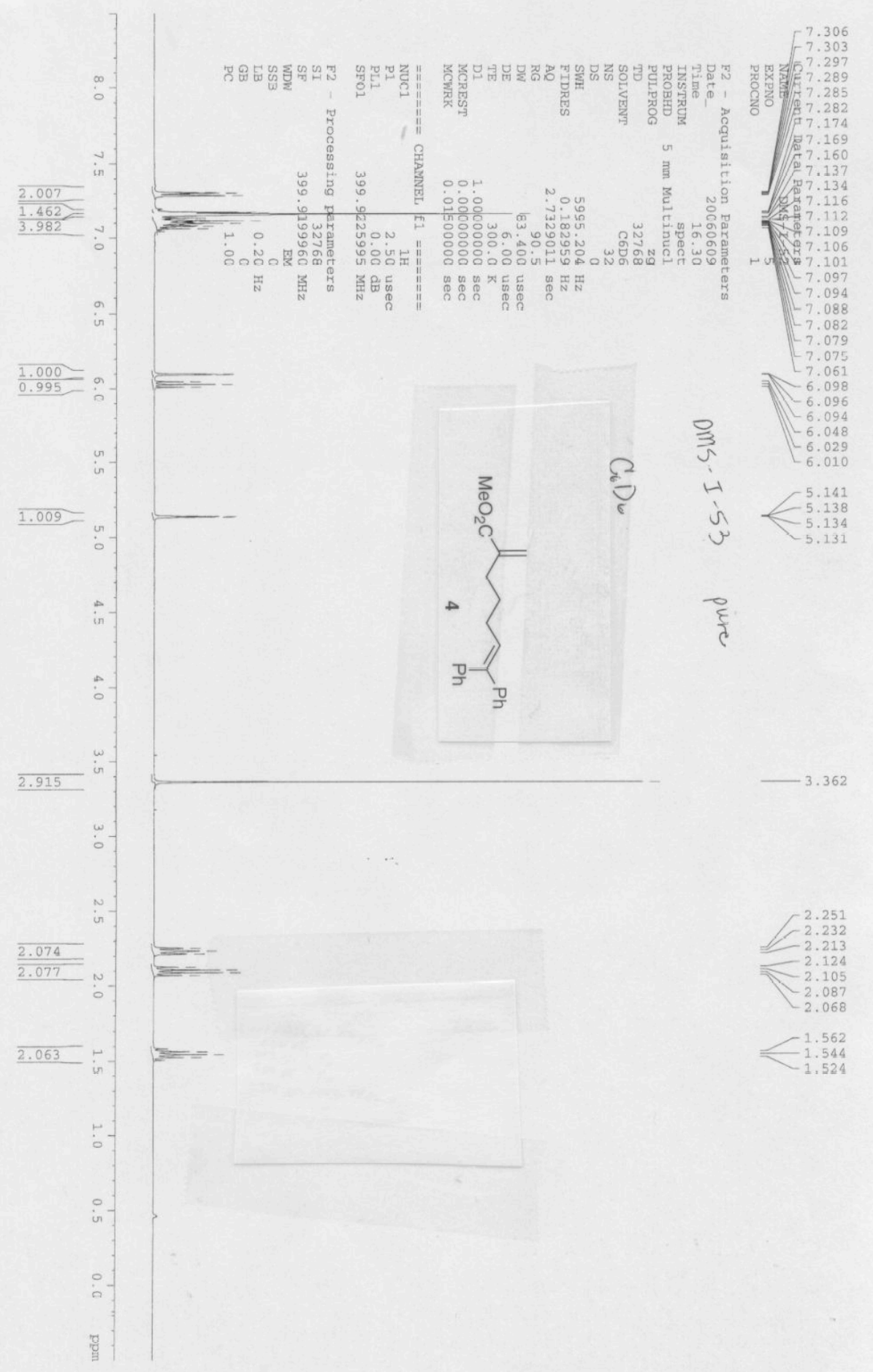




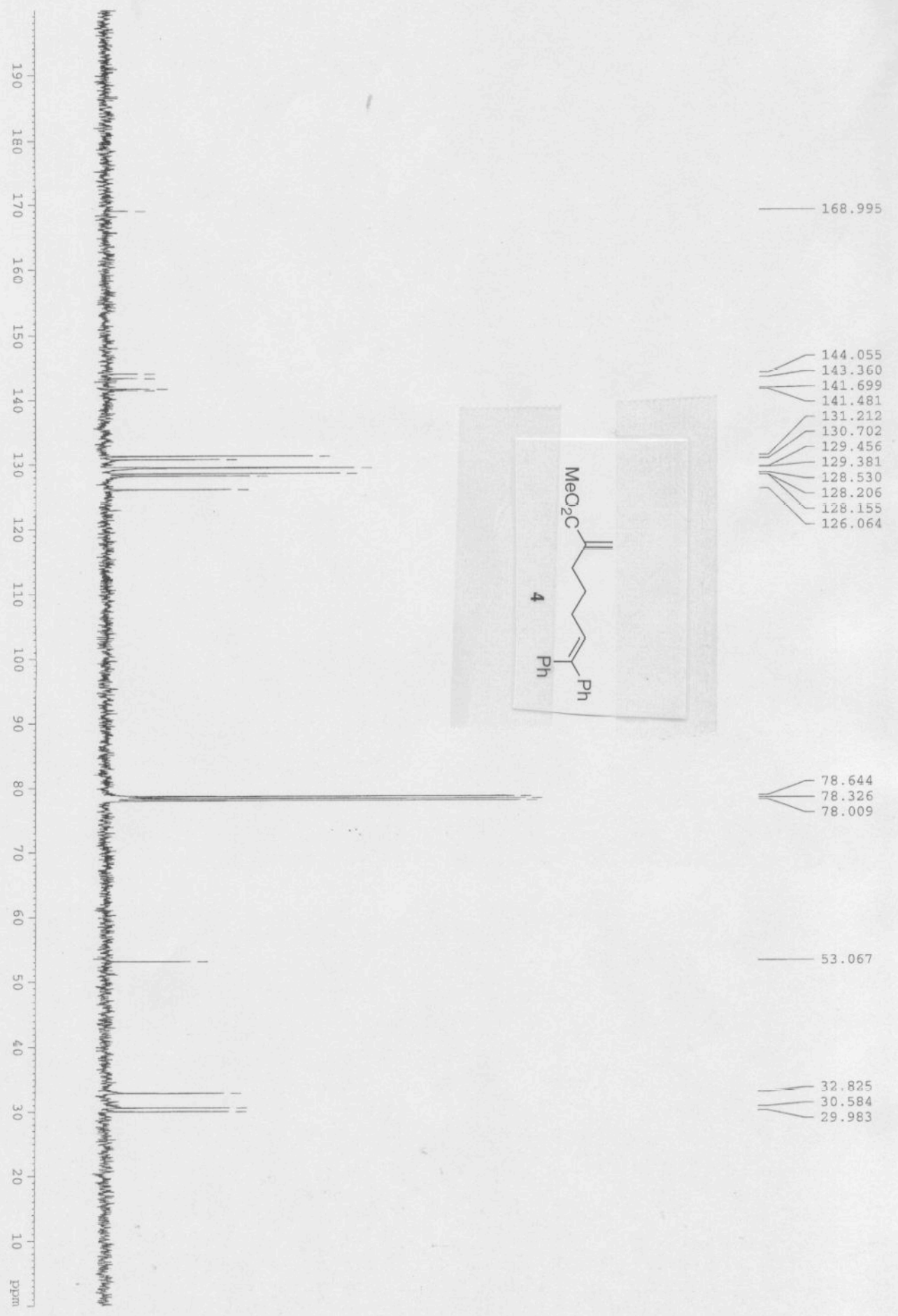




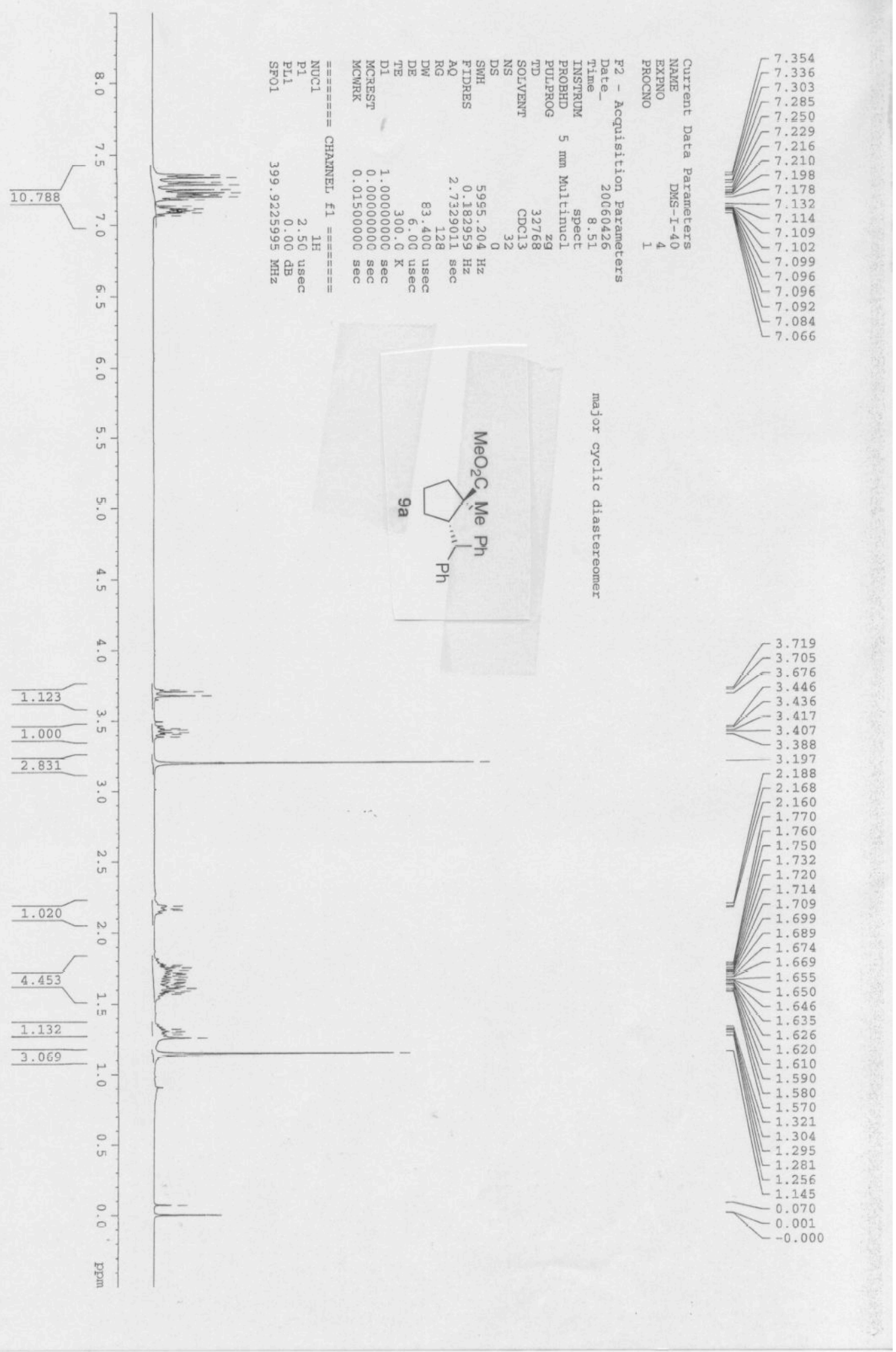




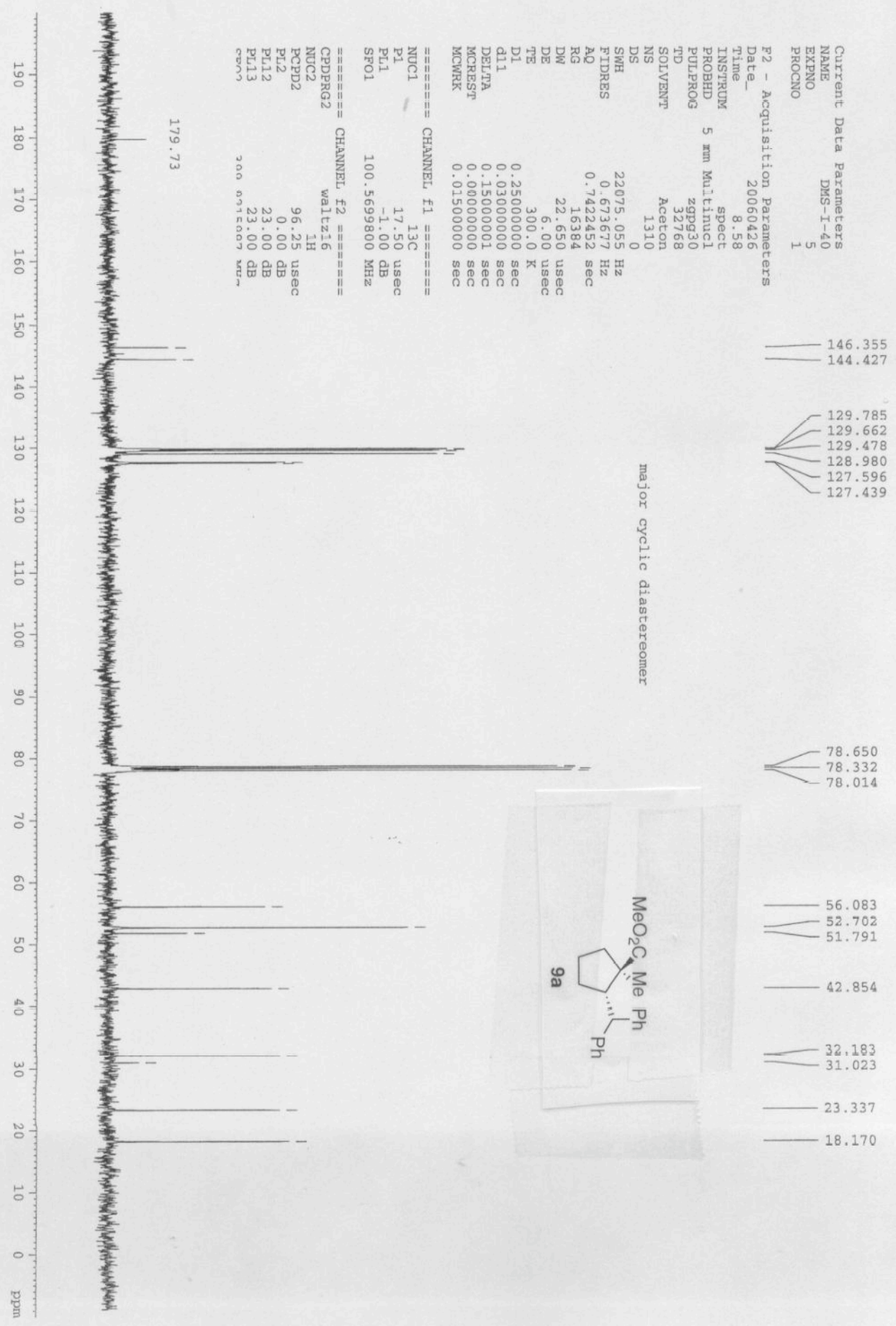




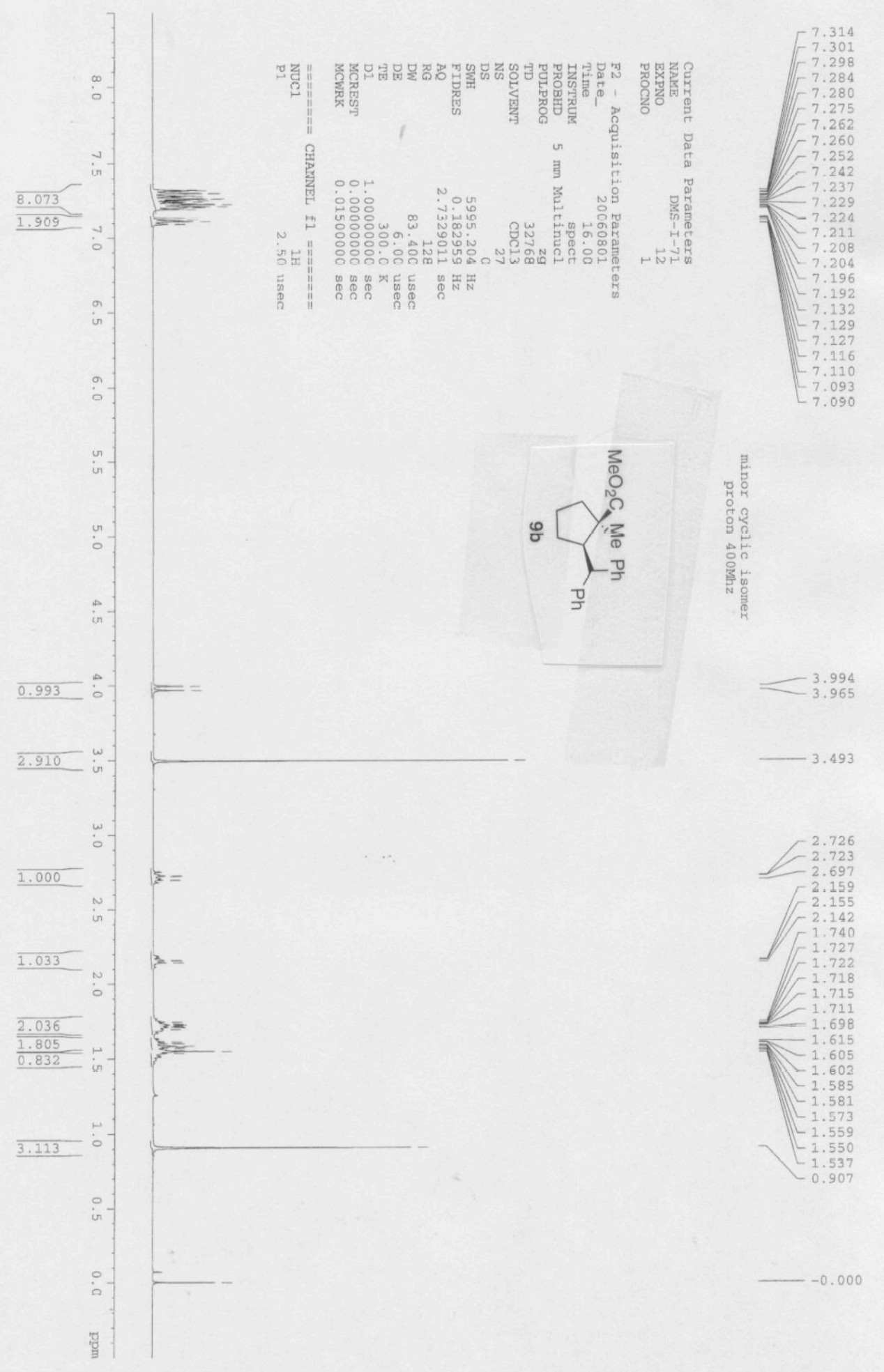




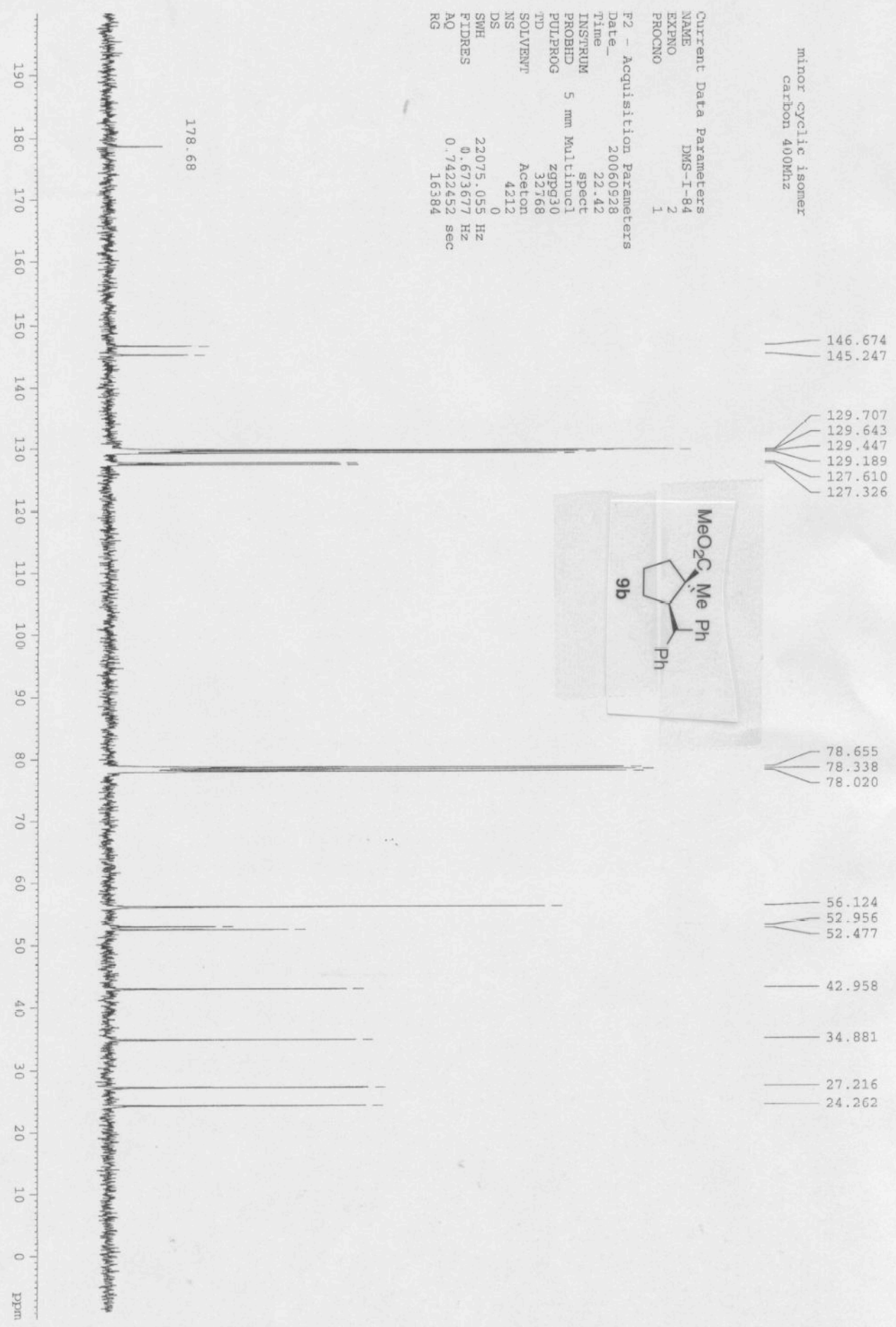




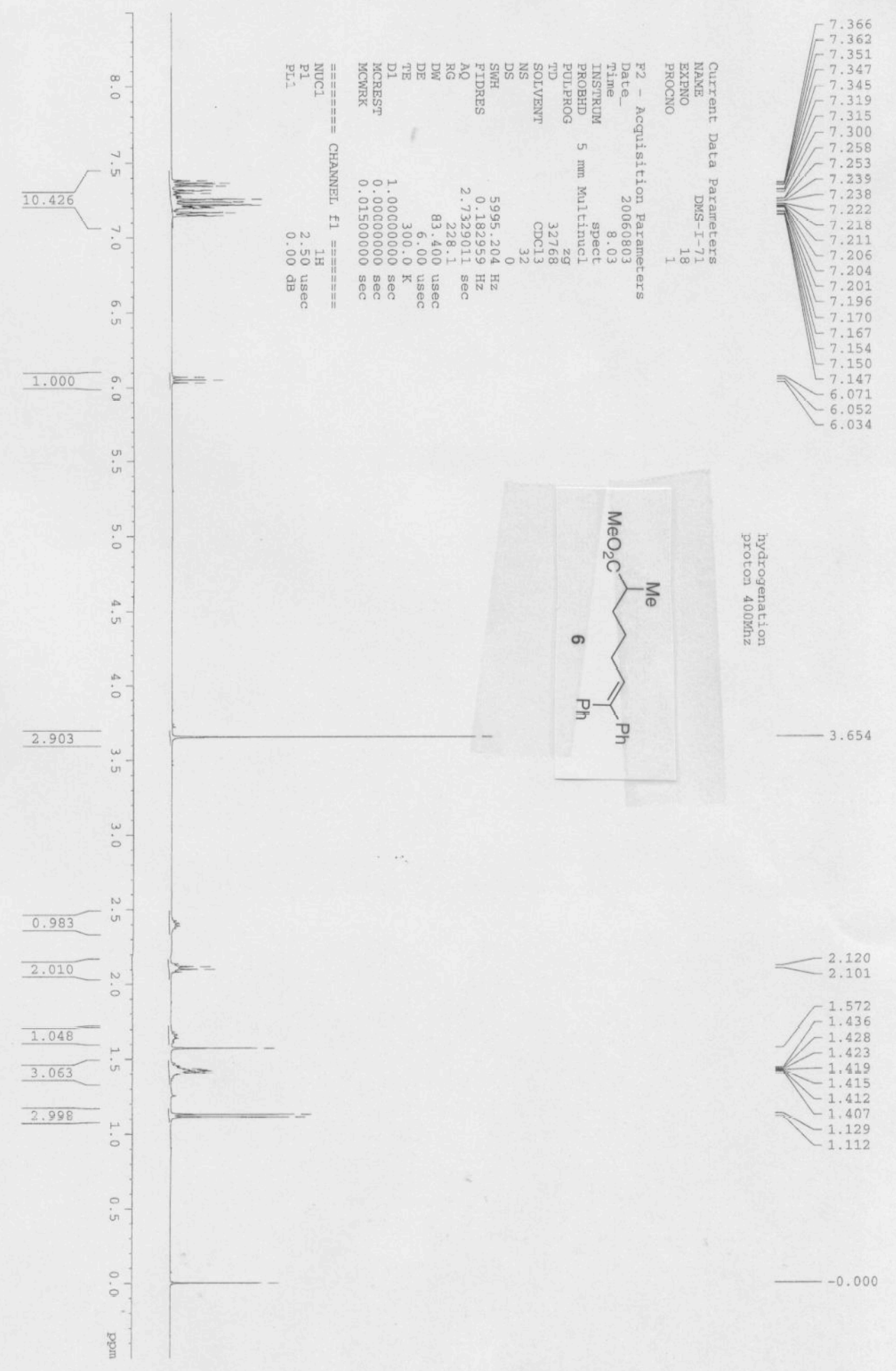




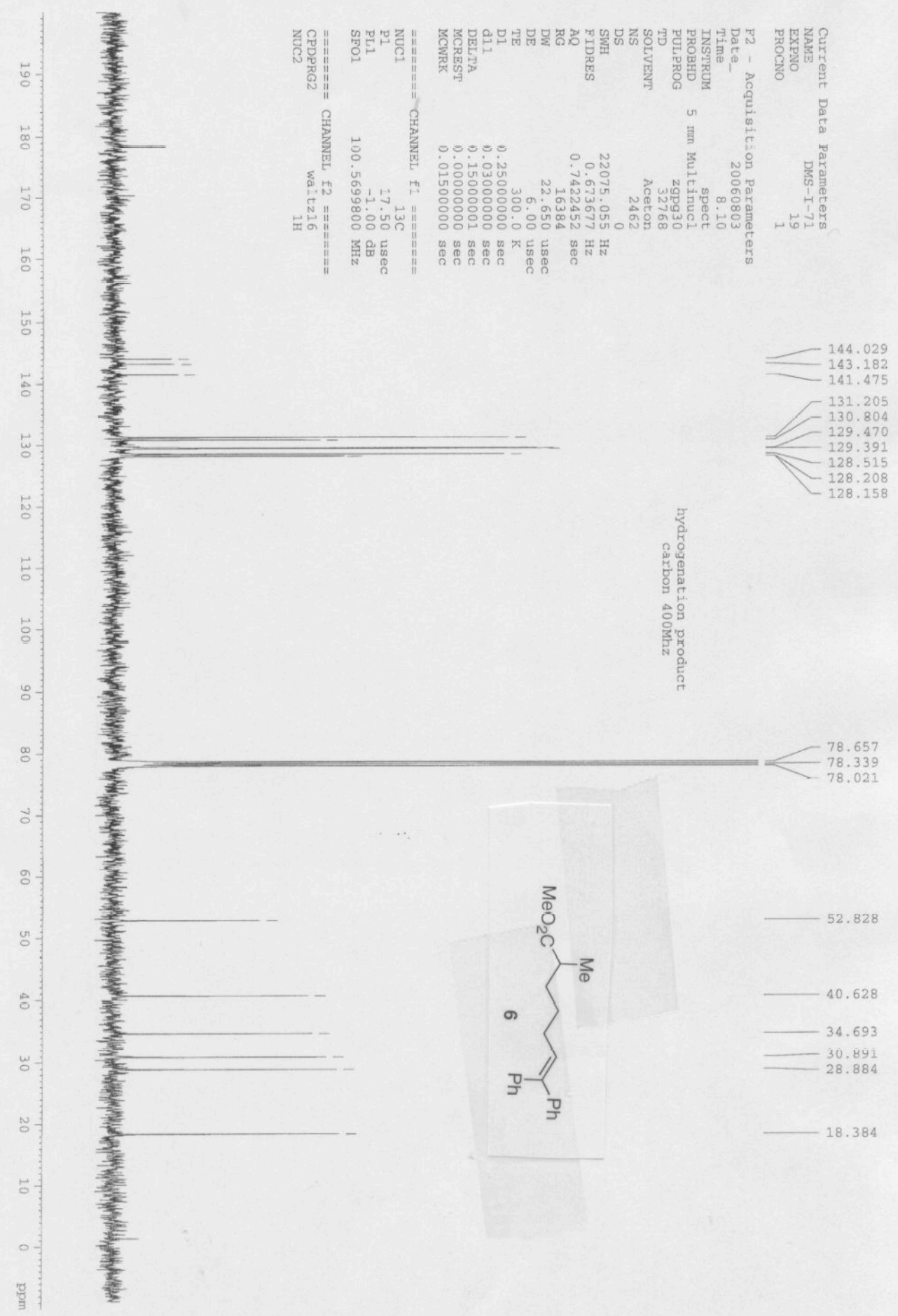




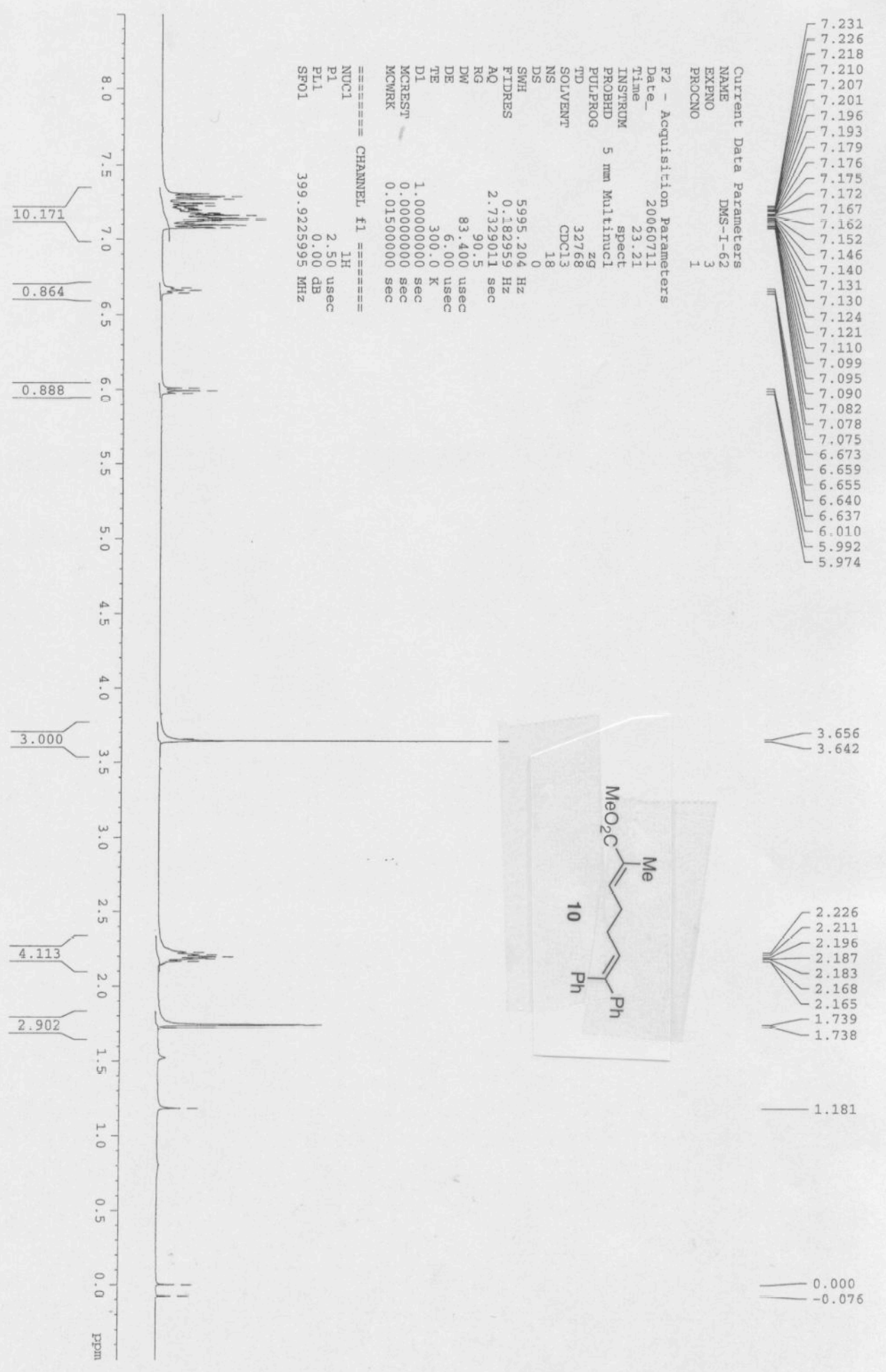




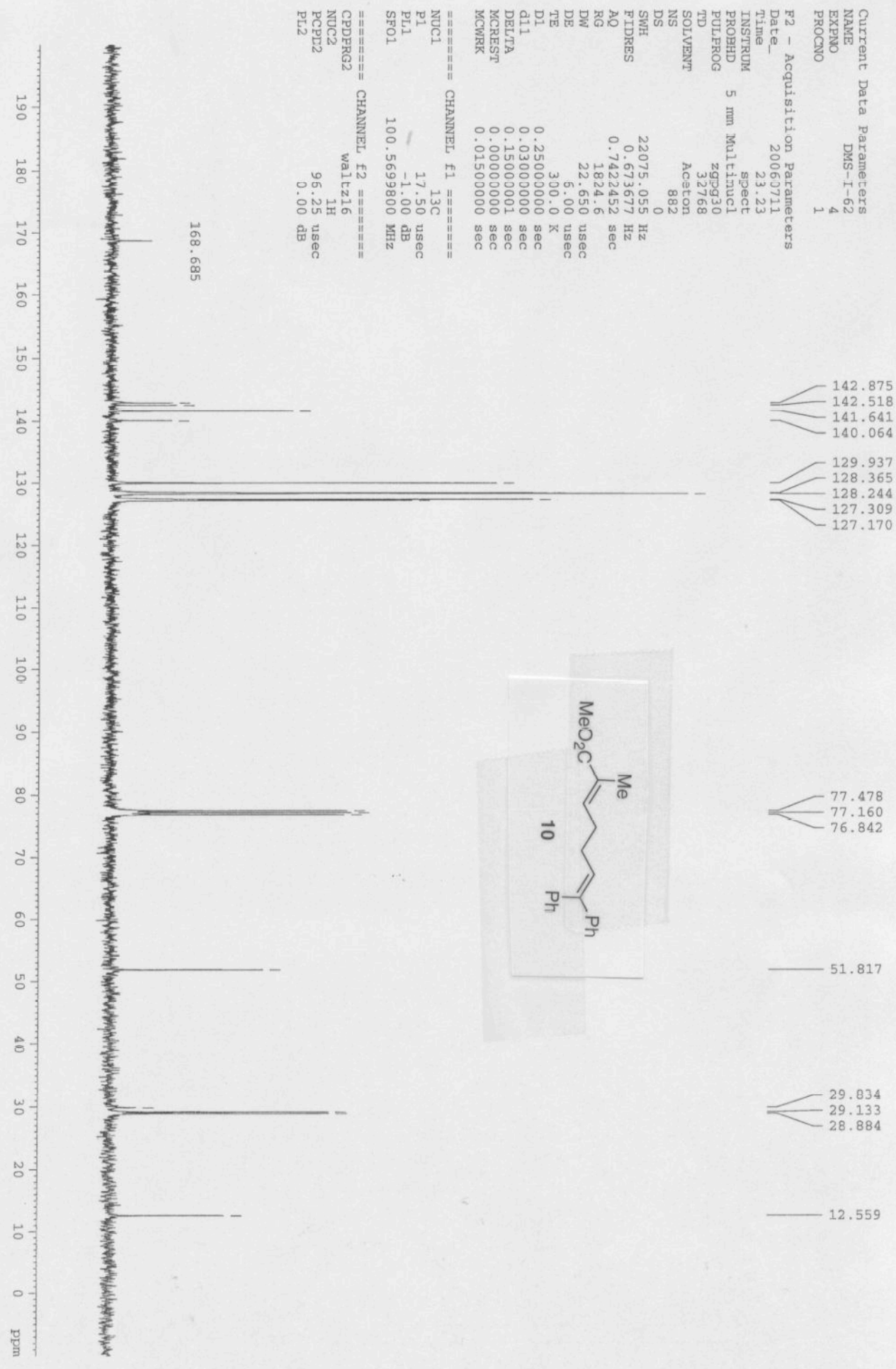




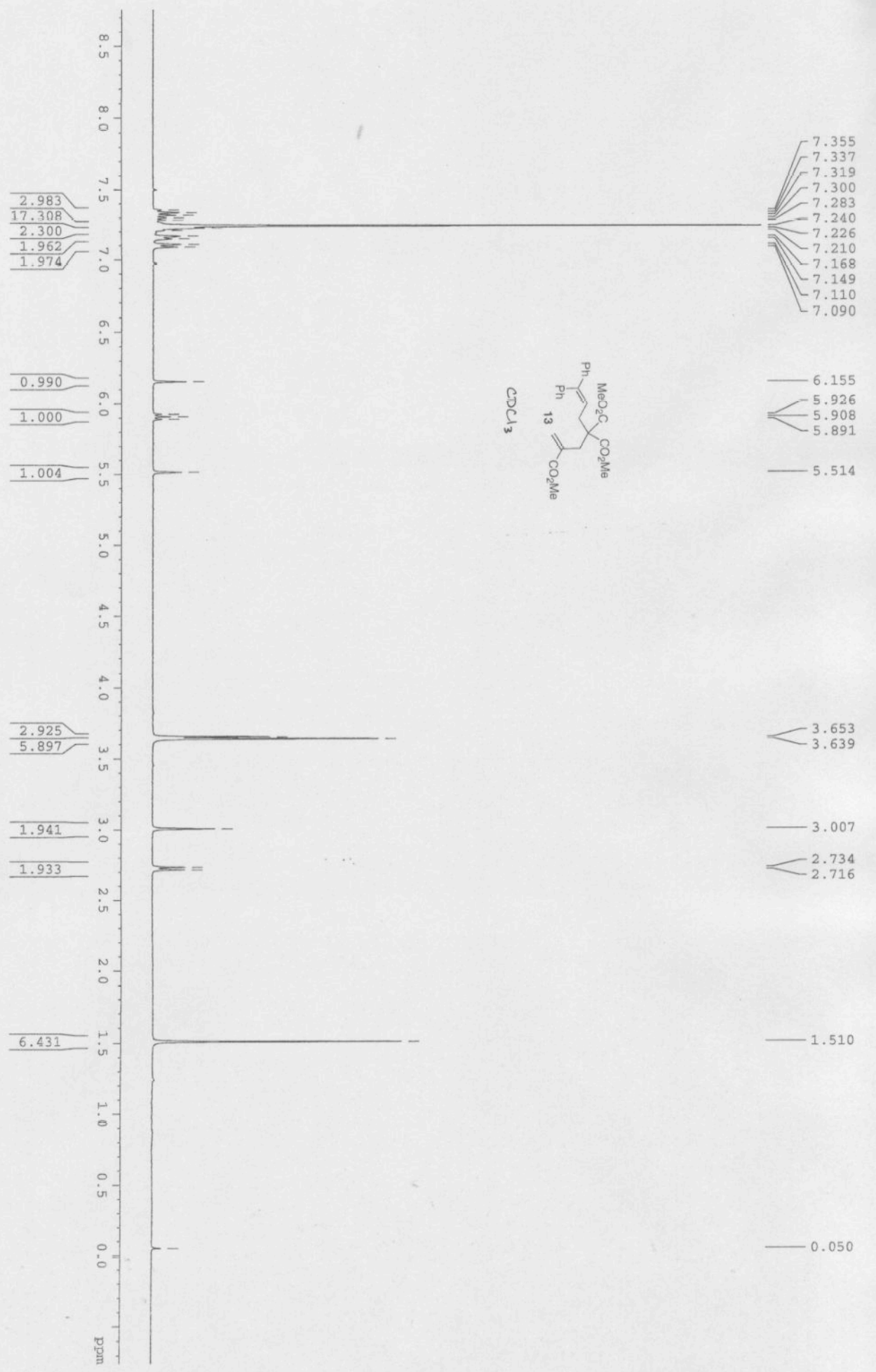




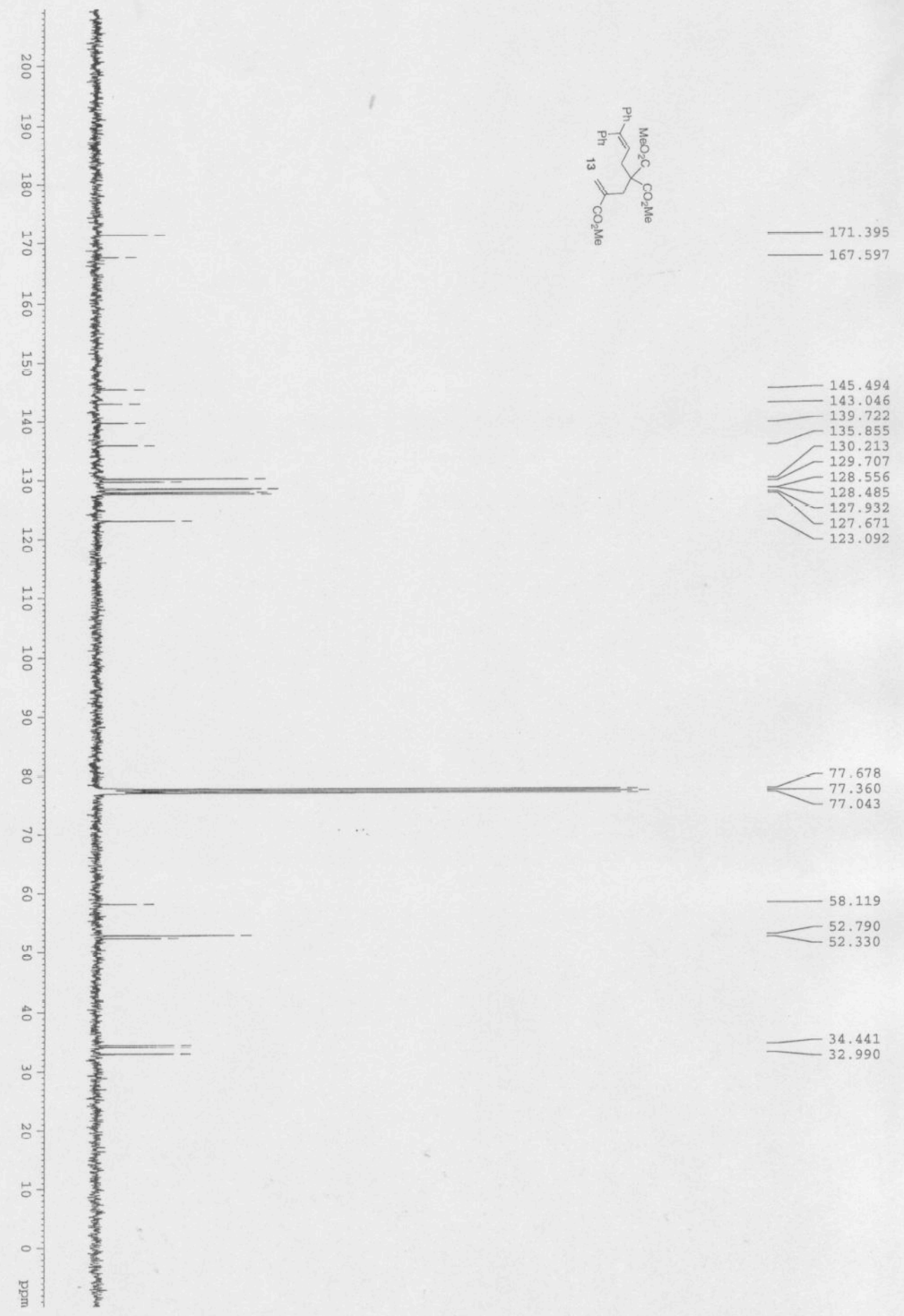




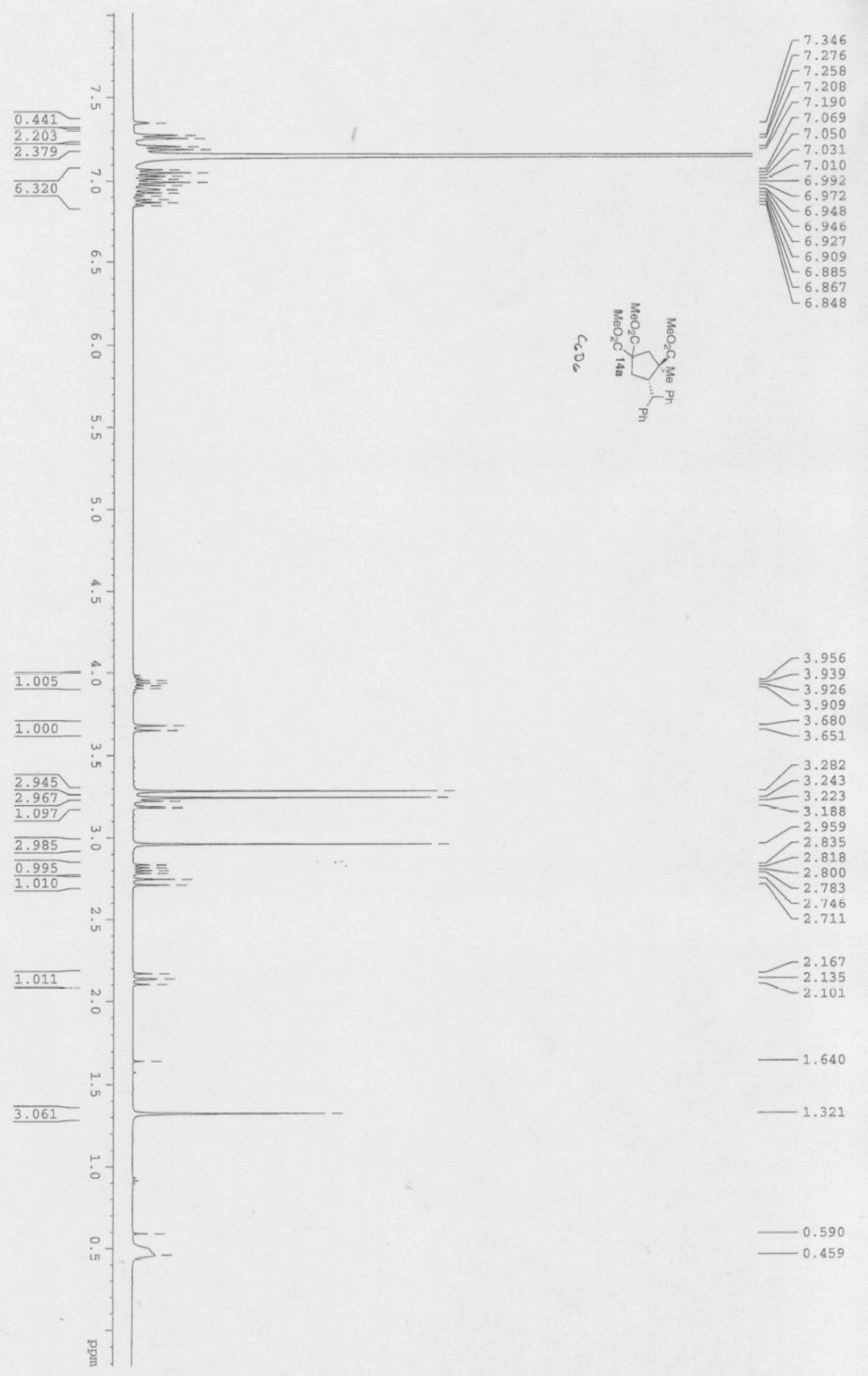




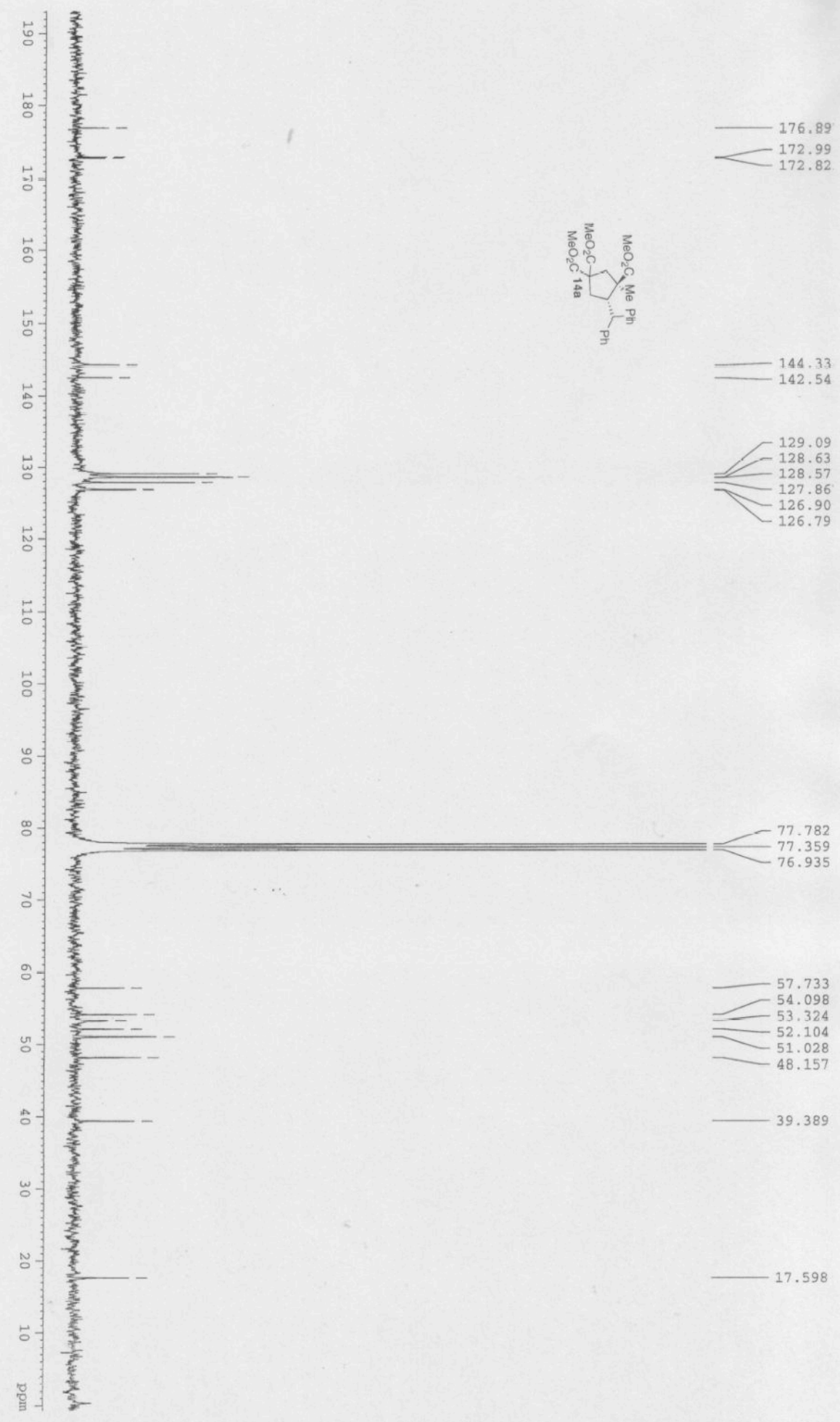




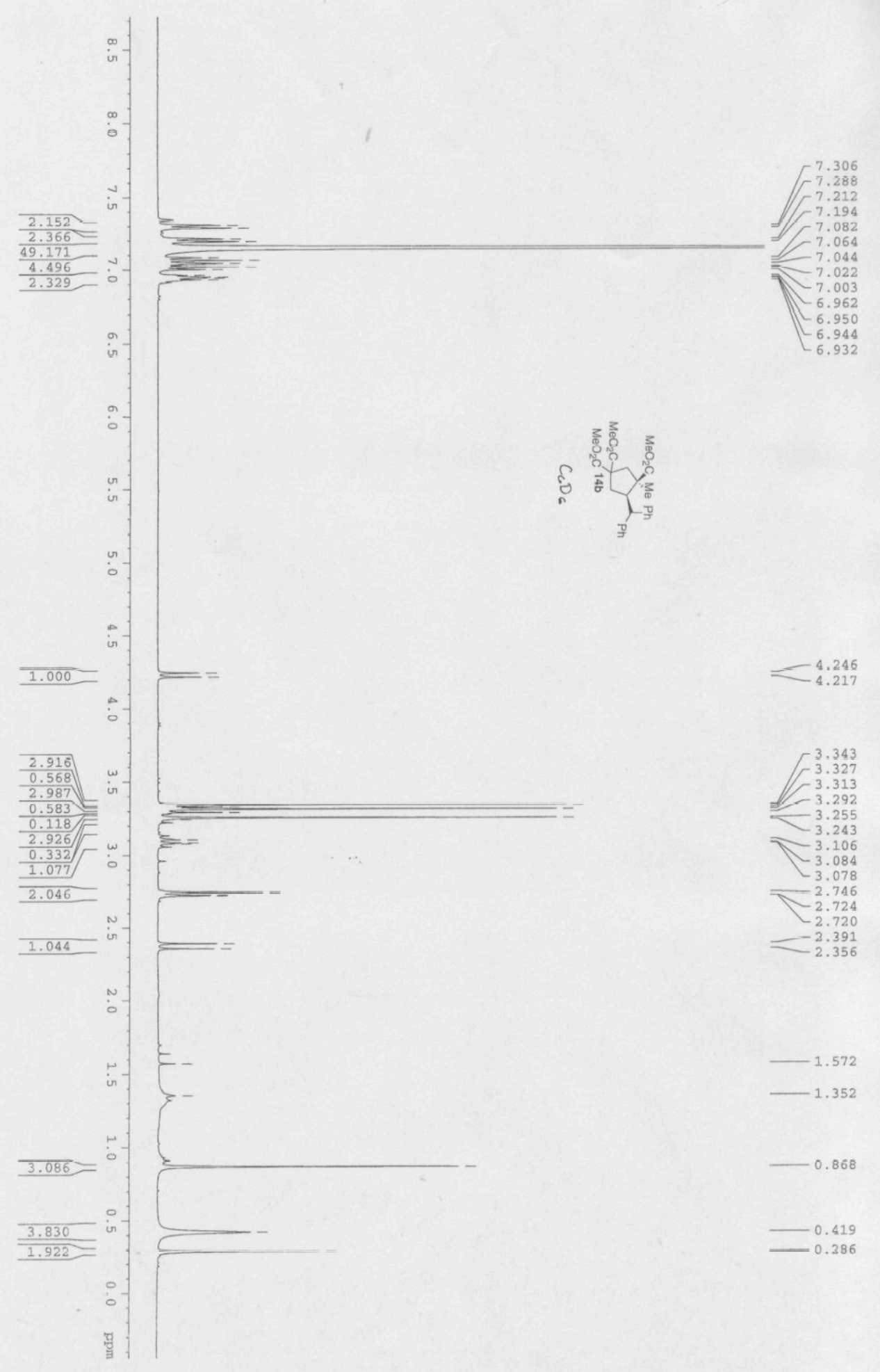




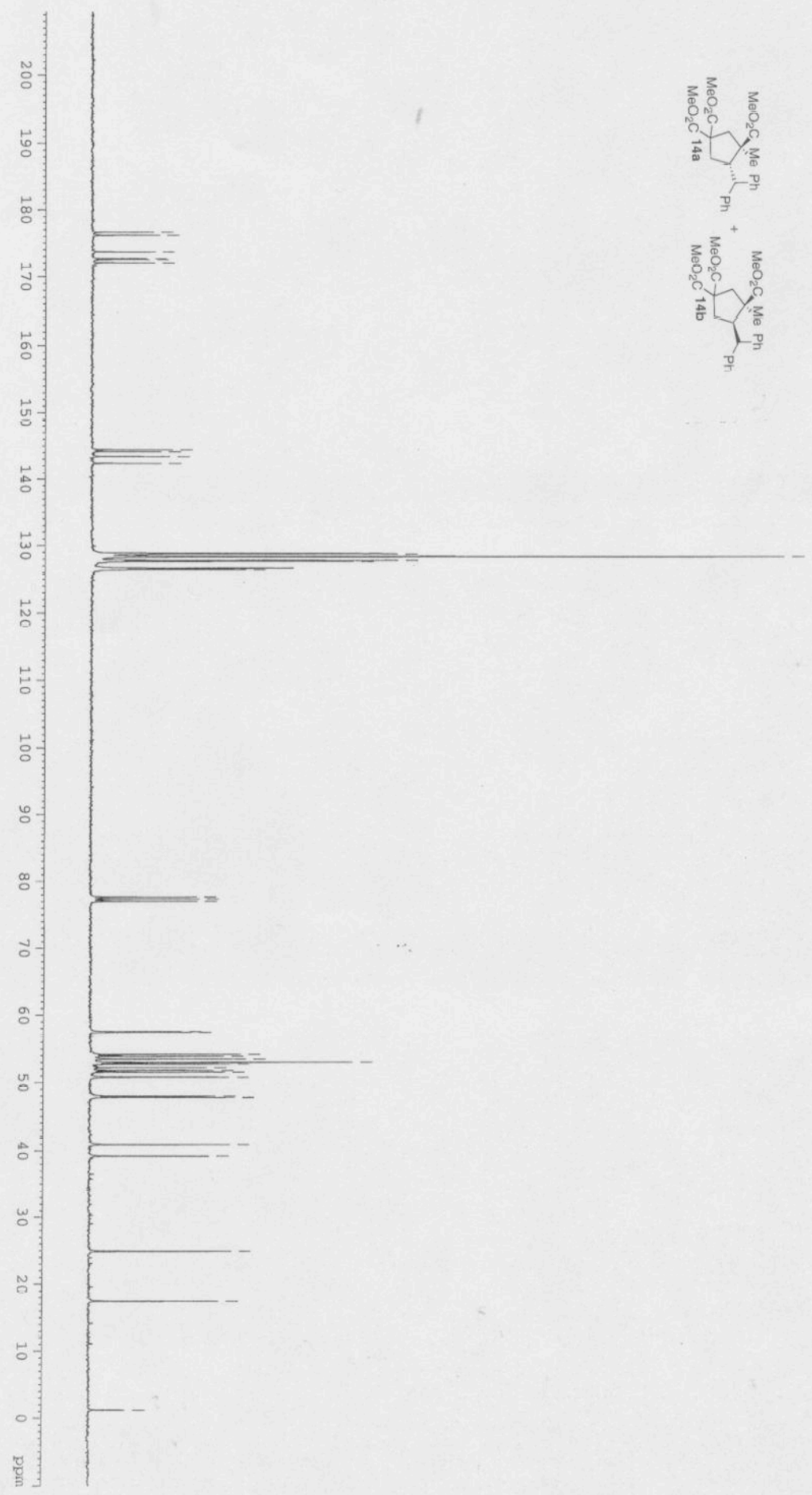

$-144.458$

$-144.188$

143.440

$-128.879$

128.879
-128.794

$-128.652$

$-128.396$

$\begin{array}{r}128.360 \\ \hline\end{array} 127.833$

$-127.833$

127.633
-126.696

$-126.669$

-126.588
-126.470

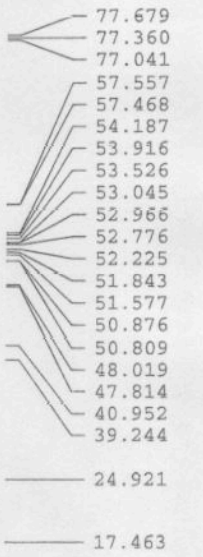

1.201 